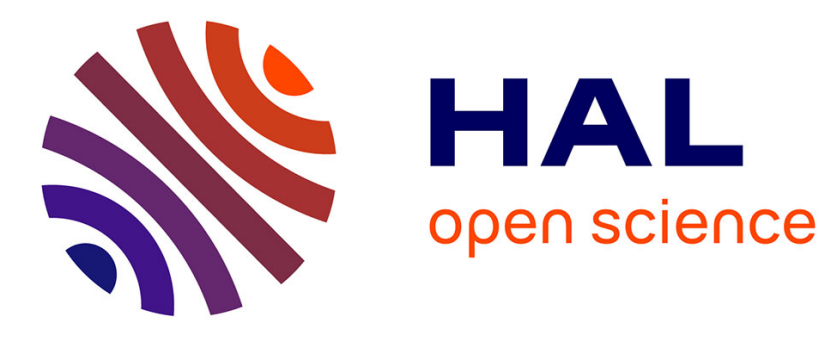

\title{
Models for Miridae, a cocoa insect pest. Application in control strategies
}

Myriam Djoukwe Tapi, Leïla Bagny Beilhe, Samuel Bowong, Yves Dumont

\section{To cite this version:}

Myriam Djoukwe Tapi, Leila Bagny Beilhe, Samuel Bowong, Yves Dumont. Models for Miridae, a cocoa insect pest. Application in control strategies. Mathematical Methods in the Applied Sciences, 2018, 41 (18), pp.8673-8696. 10.1002/mma.5063 . hal-01860823

\section{HAL Id: hal-01860823}

\section{https://hal.inria.fr/hal-01860823}

Submitted on 21 Apr 2021

HAL is a multi-disciplinary open access archive for the deposit and dissemination of scientific research documents, whether they are published or not. The documents may come from teaching and research institutions in France or abroad, or from public or private research centers.
L'archive ouverte pluridisciplinaire $\mathbf{H A L}$, est destinée au dépôt et à la diffusion de documents scientifiques de niveau recherche, publiés ou non, émanant des établissements d'enseignement et de recherche français ou étrangers, des laboratoires publics ou privés. 


\title{
Models for Miridae, a Cocoa insect pest. Application in control strategies. $^{*}$
}

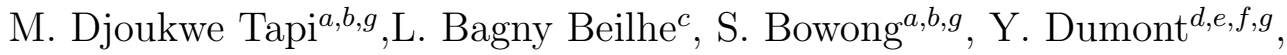 \\ ${ }^{a}$ The University of Douala, Department of Mathematics and Computer Science, Douala, Cameroon, \\ ${ }^{b}$ UMI 209 IRD/UPMC UMMISCO, University of Yaounde I, Cameroon, \\ ${ }^{c}$ CIRAD, UR Bioagresseurs, Regional Direction of CIRAD for Central Africa, Yaounde, Cameroon, \\ ${ }^{d}$ CIRAD, Umr AMAP, Pretoria, South Africa, \\ ${ }^{e}$ AMAP, Univ Montpellier, CIRAD, CNRS, INRA, IRD, Montpellier, France, \\ ${ }^{f}$ University of Pretoria, Department of Mathematics and Applied Mathematics, Pretoria, South Africa, \\ ${ }^{g}$ EPITAG, LIRIMA, France
}

August 24, 2018

\begin{abstract}
Cocoa mirid, Sahlbergella singularis, is one of the major pests of cocoa in West Africa. It is responsible of several damages in plots. In this paper, we mainly study the dynamics of this pest and its control. Based on biological and ecological partial knowledge, we develop two cooperative mathematical models that aim to describe the time dynamics of the cocoa mirids. We first develop a cooperative stage-structured model, derived qualitative results. Then, assuming that all parameters are or not periodic, we obtain conditions that allow the persistence or not of the population along the year. Then, we derive a two-stage cooperative time-delay model, with two delays, that takes into account the nymph maturation period and the females maturation period. A sensitivity analysis is provided for both models. We illustrate our theoretical results with numerical simulations and show that the delayed system provides the best results compared to real observations. Finally, we focus on chemical control, that is commonly used in Cameroon, and compare it to a new biological control, mixing mating disrupting and trapping. We discuss the results and provide future perspectives based on this work.
\end{abstract}

Keywords: Sahlbergella singularis; cocoa pest; mathematical models; delays; pest control; simulation.

\section{Introduction}

Cacao is a cash crop mainly cultivated in African countries (Ivory Coast, Ghana, Nigeria and Cameroon) that ensures around $72 \%$ of the world production [ICCO 2016]. Cocoa (Theobroma ca$c a o$ ) is essential for the livelihood of millions of small producers in Africa especially in Cameroon [2]. African production of cocoa is seriously impacted by two important diseases the Cocoa Swollen Shoot Virus (CSSV) and the black pod rot (up to 80-90\% losses) [28] and by the damage caused by two pests: Sahlbergella singularis and Distantiella theobroma known as mirid bugs or cacao capsids ([10]; [14]). These species, which originate from the forests of central Africa, have very similar

\footnotetext{
*corresponding author: Yves Dumont. email: yves.dumont@cirad.fr
} 
life histories and regularly live together in cacao-based agroforestry systems [13]. In Cameroon, Sahlbergella singularis is nowadays the most common and the most harmful species for the production. Mirid bugs feed preferentially on Theobroma cacao but other species like Cola nitida, Ceiba pentandra are known to be alternative hosts when cacao resource is unavailable. The immature (five instars) and adult stages of these sucking insects used to feed on the sap of young semi-lignified branches, on plant tissues by injecting a digestive saliva, on buds and on fruits [46, 18, 15, 34]. Mirid damage on the pod are relatively superficial since the pods cortex is very thick. In general mirids attacks on the pods are characterized by black dots (feeding lesion) around the peduncle of mature pods. Indeed, there is no obvious link between the intensity of feeding lesions on mature pods and an impact on beans quality [47].Very few losses are noted at this stage. However, lesion caused to young fruit (less than $10 \mathrm{~cm}$ and less than 2 months old), called cherelle, increase fruit abortion and impact directly the ongoing production [47]. This fruit stage is vulnerable to mirids attacks. Indeed, the most harmful damage that is also the less obvious to quantify is the damage caused to cocoa vegetative growth parts. Those lesions prevent sap circulation favouring leaves fall and branches death that is characteristic of mirids attacks. Extensive feeding by mirids on branches results in the degradation of the canopy of discrete groups of trees which can be up to one hundred and are referred as mirid pockets. Mirid pockets are generally located in the sunniest areas of plantations [15]. The impact of mirids on cacao tree is a long term impact as cacao is a perennial plant which can produce for more than 20 years. Damage of mirid bugs on the cocoa tree are cumulated over time and can lead to premature ageing of plantations and to the rapid death of the most severely damaged trees [17, 46, 27]. Losses due to mirids are difficult to estimate, but can reach $30-40 \%$ of potential production $([1,8])$ depending on the system management strategy. In fact, mirids attacks are known to be the most harmful in full sun plantations. In multi-strata and highly diversified $[22,32,45]$ cacao based agroforestry system as the one that is widespread in Cameroon, shade management is a relevant option to control mirid population [15]. But shade management is a long term process that is sometimes difficult to set up for the farmers given antagonistic effects on black pod disease. Whatever the type of system considered, synthetic insecticides of the neonicotinoid family, such as $\lambda$-cyhalothrine and Imidacloprid $[25,10]$, are still the main input used to control these pests [37]. Since 1970, the economic threshold for phytosanitary intervention has been fixed at 0.7 mirids/tree in Cameroon [15] and 0.6 mirids/tree in Ghana [40]. These indicators based on mirid populations are however difficult to evaluate regarding the ecology of the species. In fact, it is chalenging to count mirids individuals (immatures and adults) on the field since they used to hide during the day to avoid direct light. It is likely a relatively low level of mirid population can cause important damage in the plantation. Mirids do not pullulate in the plantations even during the peak period. Due to controversial effects of chemical insecticide use, alternative cocoa pest control methods have been developed including cultural management, varietal management [21], as well as semio-chemical management, using synthetic sexual pheromone traps [35] or the use of plant extracts as pesticides [10]. Considering the difficulty to estimate mirid population and to obtain long-term data on the field, the mathematical approach appears as the most relevant option to forecast the efficiency of control strategy. In that sense, the aim of this work is to develop some (generic) mathematical models of mirid population to better predict its time evolution in a plot under different management strategies. A first compartmental model, with constant or periodic parameters, is developed based on the mirid life cycle. We make theoretical analysis, a sensitivity analysis and provide numerical simulations. Then, we develop a second model, a time delayed model, based on the previous one, in order to better take into account the 
developmental or maturation time in some stages. After a brief theoretical analysis, we provide numerical simulations without and with control efforts, and we discuss the results.

The paper is organized as follows. In section 2, we formulate a first ODE model considering the resource as constant or periodic function: for this model, we make a theoretical study (its global dynamical properties), a sensitivity analysis and numerical simulations. In section 3, we formulate a delayed model. After a brief study, we do some numerical simulations and we compare with the previous model without delay. In section 4, we compare chemical and biological controls and discuss their efficiency. Finally, in Section 5 we conclude and propose several experimental and theoretical studies in order to improve our knowledge and extend our work.

\section{Mirids ODEs models}

In order to build the models, we recall here what we know about the biology and ecology of Mirids. The life cycle of $S$. singularis is composed of three stages: egg stage, nymph stage and adult stage that develop mainly on pods either on shoots. The eggs are individually inserted into the host plant tissues [33] principally in the cortex of pods and sometimes under the bark of young shoots [26]. The incubation period of eggs is on average 15 days with a minimum of 9 days [48] and a maximum of 21 days [31] before reaching nymph stage. Mirid S. singularis has a very long life cycle (eggs to adults). It is on average 40 days with a minimum of 36 days [12] and a maximum of 50 days [13]. The percentage of hatching eggs is globally $96.53 \%$ as the eggs are protected in the pods cortex. During the five nymph instars, the individuals move within a cacao tree by walk to feed on the pods and shoots. The nymphs are able to feed just after the eggs hatching. Twenty five days are needed to complete the nymph development considering 5 days in average per instar [13]. Globally, the total nymph survivorship is around $68 \%$ [11]. The average daily rate of survival of the nymphs (considering all the five instars) was estimated around $98.5 \%$ (estimated using biological data). At the emergence there is on average one female for 0.71 male [13] (this gives a sex ratio $r=1 / 1.71 \approx 0.58$; in fact, sex ratio varies between 0.5 and 0.6 ). Females $S$. singularis mate with one male 6 to 10 days after their emergence. The first eggs are observed in average 10 days after emergence, so 4 to 8 days after mating (estimated using biological data). Indeed, after this mating period females are considered as mature. On average $72.1 \%$ of immature females become mature females [12]. After the emergence, adults (males and females) fly from one cocoa tree to another ensuring the spatial dispersion of the individuals and causing the spatial distribution of the damage in the plots. It is likely the females do not lay all their eggs on the same pod. The average fecundity per female is around 50.7 larvae or 52.5 eggs and the fecundity period lasts on average 16 days $[46,27,48,7,16]$. The daily survival of mirids adult is around respectively $98.14 \%$ for immature females, $92.8 \%$ for mature females and $93 \%$ for males (estimated thanks to $[7,31,48,13,46])$. On average, 50 to 60 days are needed to obtain a new generation of mirids [13]. This long life period for an insect to grow is a key factor for the dynamic of mirid populations.

Mirids population dynamics varied greatly during the year. Density of population is likely to be influenced by pods availability on the trees and by external conditions like weather [15]. The mirid population is low on cocoa during the period from February to March. From June to July, the populations start to grow more or less rapidly. The peak of the population appears between

September and November when the pods are almost mature [13, 38, 7, 39]. It is also assumed that unfavourable climatic conditions (high temperature and low pluviometry) can cause declining 
fertility of females and increased mortality of individuals [15] that lead to lower mirid populations observed in plantations from November-December to June.

We now formulate a model based on the life cycle of $S$. singularis, summarized in Fig. 1 . We use a stage structured model. We consider three main stages in the development of the mirid: the egg stage $(E)$, the nymph stage $(L)$ (nymphs and pupae) and the adult stage, subdivided into immature female $\left(F_{1}\right)$, mature female $\left(F_{2}\right)$ and male $(M)$. According to the flow diagram given in

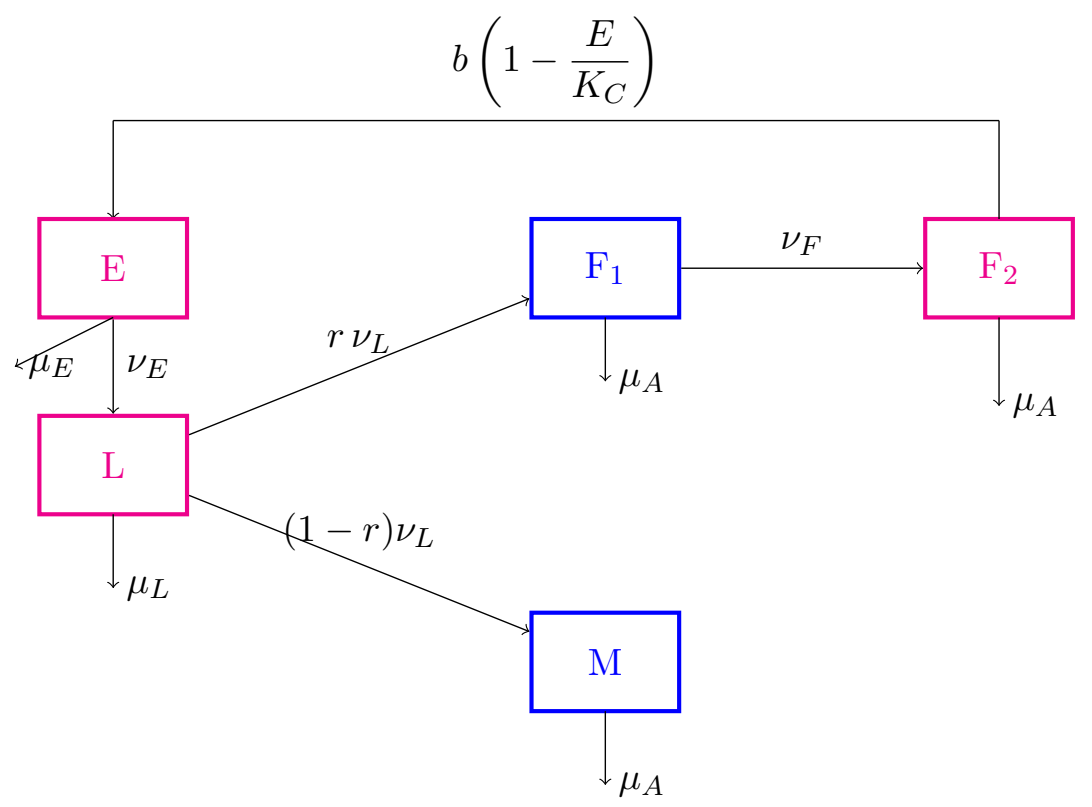

Figure 1: Life cycle of Sahlbergella singularis

Fig. 1, we derive the following ODES model:

$$
\left\{\begin{array}{l}
\dot{E}=b F_{2}\left(1-\frac{E}{K_{C}}\right)-\left(\nu_{E}+\mu_{E}\right) E \\
\dot{L}=\nu_{E} E-\left(\nu_{L}+\mu_{L}\right) L \\
\dot{F}_{1}=r \nu_{L} L-\left(\nu_{F}+\mu_{A}\right) F_{1} \\
\dot{F}_{2}=\nu_{F} F_{1}-\mu_{A} F_{2} \\
\dot{M}=(1-r) \nu_{L} L-\mu_{A} M
\end{array}\right.
$$

with non-negative initial conditions:

$$
\begin{cases}E(0)=E^{0}, & L(0)=L^{0}, \quad F_{1}(0)=F_{1}^{0}, \\ F_{2}(0)=F_{2}^{0}, & M(0)=M^{0} .\end{cases}
$$

The biological parameters are described as follows: $r$ is the sex ratio; $b$ is the mean number of eggs laid by an adult female mirid per day that have emerged as nymphs, $K_{C}$ is the maximal carrying capacity related to the mean daily number of pods per area (ha), $\mu_{E}, \mu_{L}$ and $\mu_{A}$ represents respectively the eggs, nymphs and adults daily mortality rate, $\nu_{E}$ and $\nu_{L}$ are respectively the transition rate from the egg to nymph stage and the nymph to adult stage; $\left(1 /\left(\nu_{E}+\mu_{E}\right)\right.$ and $\left(1 /\left(\nu_{L}+\mu_{L}\right)\right.$ are respectively the mean time a mirid stays in the egg and nymph stage (measured in days); $\nu_{F}$ is the transition rate from the immature female stage to mature female stage; $1 /\left(\nu_{F}+\mu_{A}\right)$ is the mean lifespan of an immature female mirid, measured in days. 
The non linear term $r b F_{2}\left(1-\frac{E}{K_{C}}\right)$ is related to a specific behaviour of some insects species, like Aedes mosquito [19, 20], and also mirids, known as skip-oviposition behaviour. Indeed, according to expert's knowledge, mirids (S. singularis) are able to select their breeding sites. Some cocoa trees, particularly suitable for nymph development, show greater damage, which leads to the degradation of the foliage and the formation of orthotropic (or greedy) shoots [11]. Thus, if breeding sites, in a given area, already contain a lot of eggs, then females will not deposit eggs or only very few. That is why, the oviposition rate $r b F_{2}$ is limited by the available space in breeding sites, $\left(1-\frac{E}{K_{C}}\right)$, which implies that the birth rate in the eggs compartment is modeled by the non linear term $r b F_{2}\left(1-\frac{E}{K_{C}}\right)$. Table 1, page 5, summarizes the parameters and their biological meaning.

\begin{tabular}{|c|l|l|}
\hline Parameters & Biological significance & Unit \\
\hline$b$ & Mean number of eggs laid by a mature female & days $^{-1}$ \\
\hline$K_{C}$ & Maximal carrying capacity related to the mean daily number of pods per ha & \\
\hline $1 / \nu_{L}$ & Duration of the development of nymphs & days \\
\hline $1 / \nu_{F}$ & Time necessary for an immature female to become mature & days $^{-1}$ \\
\hline$\mu_{L}$ & Mortality of nymphs & days $^{-1}$ \\
\hline$\mu_{A}$ & Mortality of adults & days $^{-1}$ \\
\hline$\mu_{E}$ & Mortality of eggs & days $^{-1}$ \\
\hline $1 / \nu_{E}$ & Time necessary for an egg to become nymph & days \\
\hline
\end{tabular}

Table 1: Parameters of model (1).

The right-hand side of system (1) is continuously differentiable $\left(\mathcal{C}^{1}\right)$. Then, using the CauchyLipschitz theorem, system (1) has a unique maximal solution. If the initial data are in $\mathbb{R}_{+}^{5}$, the solutions stays in $\mathbb{R}_{+}^{5}: E=0, L=0, F_{1}=0, F_{2}=0$ and $M=0$ are vertical and horizontal null lines respectively. Thus, the trajectories can not cut these axes: so model system (1) is biologically well posed. It is straightforward to show that the compact

$$
\begin{array}{r}
\Omega=\left\{\left(E, L, F_{1}, F_{2}, M\right) \in \mathbb{R}_{+}^{5} ; E(t) \leq K_{C}, L(t) \leq \frac{\nu_{E} K_{C}}{\nu_{L}+\mu_{L}} F_{1}(t) \leq \frac{r \nu_{L} \nu_{E} K_{C}}{\left(\nu_{L}+\mu_{L}\right)\left(\mu_{A}+\nu_{F}\right)},\right. \\
\left.F_{2}(t) \leq \frac{r \nu_{L} \nu_{F} \nu_{E} K_{C}}{\mu_{A}\left(\nu_{L}+\mu_{L}\right)\left(\nu_{F}+\mu_{A}\right)} \text { and } M(t) \leq \frac{(1-r) \nu_{L} \nu_{E} K_{C}}{\mu_{A}\left(\nu_{L}+\mu_{L}\right)}\right\}
\end{array}
$$

is positively invariant by (1).

Now, we will derive some quantitative analysis of system (1). Let us consider the following threshold

$$
\mathcal{N}_{0}=\frac{r b \nu_{L} \nu_{F} \nu_{E}}{\mu_{A}\left(\nu_{E}+\mu_{E}\right)\left(\nu_{F}+\mu_{A}\right)\left(\nu_{L}+\mu_{L}\right)} .
$$

$\mathcal{N}_{0}$ represents the mean number of adults female produced by one adult female over its lifespan. It is sometimes called the basic offspring number.

Lemma 2.1 System (1) has two possible equilibria:

(i) a trivial equilibrium $X^{0}=(0,0,0,0,0)$ which always exists 
(ii) a positive equilibrium $X^{*}=\left(E^{*}, L^{*}, F_{1}^{*}, F_{2}^{*}, M^{*}\right)$, defined as follows:

$$
\begin{aligned}
& E^{*}=\frac{\left(\mathcal{N}_{0}-1\right)}{\mathcal{N}_{0}} K_{C}, L^{*}=\frac{\nu_{E}}{\nu_{L}+\mu_{L}} E^{*}, F_{1}^{*}=\frac{r \nu_{E} \nu_{L}}{\left(\nu_{L}+\mu_{L}\right)\left(\nu_{F}+\mu_{A}\right)} E^{*}, \\
& F_{2}^{*}=\frac{r \nu_{E} \nu_{L} \nu_{F}}{\mu_{A}\left(\nu_{L}+\mu_{L}\right)\left(\nu_{F}+\mu_{A}\right)} E^{*} \text { and } M^{*}=\frac{(1-r) \nu_{E} \nu_{L}}{\mu_{A}\left(\nu_{L}+\mu_{L}\right)} E^{*} .
\end{aligned}
$$

which exists when $\mathcal{N}_{0}>1$.

It is straightforward to verify that system (1) is a cooperative system [44]. We briefly recall its definition. Let us consider an $n$-dimensional autonomous differential system:

$$
\dot{x}=f(x), \quad x(0)=x_{0}
$$

where $\Omega \subseteq \mathbb{R}^{n}$ is an open subset, and $f$ is a given vector function, i.e $f=(f)_{i}$, such that $f: \Omega \longrightarrow$ $\mathbb{R}^{n}$ is continuous. System (6) is called cooperative if for every $i, j \in\{1,2, \ldots, n\}$ such that $i \neq j$, the function $f_{i}\left(x_{1}, \ldots, x_{n}\right)$ is monotone increasing with respect to $x_{j}$. For cooperative systems, like (6), the global asymptotic stability of an equilibrium can be studied using the following theorem:

Theorem 2.1 (see Theorem 6 [5]) Let $a, b \in \Omega$ such that $a<b,[a, b] \subset \Omega$ and $f(b) \leq 0 \leq f(a)$; where $[a, b]=\left\{x \in \mathbb{R}^{n}, a_{i} \leq x_{i} \leq b_{i}, i=1, . ., n\right\}$. Then (6) defines a (positive) dynamical system on $[a, b]$. Moreover, if $[a, b]$ contains a unique equilibrium $p$ then $p$ is globally asymptotically stable on $[a, b]$.

The dynamic of system (1) is summarized in the following theorem:

Theorem 2.2 Assume that $\left(E^{0}, L^{0}, F_{1}^{0}, F_{2}^{0}, M^{0}\right) \in \Omega$.

(i) When $\mathcal{N}_{0} \leq 1$, the trivial equilibrium $X^{0}$ is globally asymptotically stable, which means that the mirid population will dwindle until extinction, whatever the initial population.

(ii) When $\mathcal{N}_{0}>1$, the trivial equilibrium $X^{0}$ is unstable, and the positive equilibrium $X^{*}$ is globally asymptotically stable, which means that the mirid population persists.

Proof It suffices to verify the assumptions of theorem 2.1 .

(i) When $\mathcal{N}_{0} \leq 1$, model system (1) has only the trivial equilibrium $X^{0}$. By taking $a=0$ and

$$
b=\left(K_{C}, \frac{2 \nu_{E} K_{C}}{\nu_{L}+\mu_{L}} \frac{3 r \nu_{L} \nu_{E} K_{C}}{\left(\nu_{L}+\mu_{L}\right)\left(\nu_{F}+\mu_{A}\right)}, \frac{4 r \nu_{E} \nu_{L} \nu_{F} K_{C}}{\mu_{A}\left(\nu_{L}+\mu_{L}\right)\left(\nu_{F}+\mu_{A}\right)}, \frac{2(1-r) \nu_{E} \nu_{L} K_{C}}{\mu_{A}\left(\nu_{L}+\mu_{L}\right)}\right),
$$

we have $f(a)=0$ and $f(b) \leq 0$. Thus, according to Theorem 2.1 , the trivial equilibrium $X^{0}$ is globally asymptotically stable on $[0, b]$, hence on $\Omega$ when $\mathcal{N}_{0} \leq 1$.

(ii) When $\mathcal{N}_{0}>1$, there exists $\varepsilon>0$ such that $\mathcal{N}_{0}>1+\varepsilon$. Let $E_{\varepsilon}$ sufficiently small such that

$$
\begin{aligned}
& E_{\varepsilon} \leq \varepsilon, \quad L_{\varepsilon}=\frac{\nu_{E}(1+\varepsilon)}{\left(\nu_{L}+\mu_{L}\right) \mathcal{N}_{0}} E_{\varepsilon}, \quad F_{1 \varepsilon}=\frac{r \nu_{L}(1+\varepsilon)}{\left(\nu_{F}+\mu_{A}\right) \mathcal{N}_{0}} L_{\varepsilon}, \\
& F_{2 \varepsilon}=\frac{\left(\nu_{E}+\mu_{E}\right)(1+\varepsilon)^{2}}{b \mathcal{N}_{0}^{2}} E_{\varepsilon}, \quad M_{\varepsilon}=\frac{(1-r) \nu_{L}(1+\varepsilon)}{\mu_{A} \mathcal{N}_{0}} L_{\varepsilon}
\end{aligned}
$$


Let $b_{\varepsilon}=\left(E_{\varepsilon}, L_{\varepsilon}, F_{1 \varepsilon}, F_{2 \varepsilon}, M_{\varepsilon}\right)$. Then, from the right-hand side of (1) and the fact that $\mathcal{N}_{0}>1+\varepsilon$ and $K_{C} \gg 1$, we deduce:

$$
f\left(b_{\varepsilon}\right) \geq\left(\begin{array}{c}
\varepsilon\left(\nu_{E}+\mu_{E}\right)(1+\varepsilon)^{2}\left(1-\frac{1+\varepsilon}{K_{C}}\right) E_{\varepsilon} \\
\nu_{E}\left(1-\frac{1+\varepsilon}{\mathcal{N}_{0}}\right) E_{\varepsilon} \\
r \nu_{L}\left(1-\frac{1+\varepsilon}{\mathcal{N}_{0}}\right) L_{\varepsilon} \\
\nu_{F}\left(1-\frac{1+\varepsilon}{\mathcal{N}_{0}}\right) F_{1 \varepsilon} \\
(1-r) \nu_{L}\left(1-\frac{1+\varepsilon}{\mathcal{N}_{0}}\right) L_{\varepsilon}
\end{array}\right)>0 .
$$

Hence, according to Theorem 2.1, equilibrium $X^{*}$ is globally asymptotically stable on $\left[b_{\varepsilon}, b\right]$. Since $b_{\varepsilon}$ can be selected to be smaller than any $x>0$, we have that $X^{*}$ is asymptotically stable on $\Omega$ with basin of attraction $\tilde{\Omega}=\Omega \backslash\{(0,0,0,0,0)\}$. This also implies that $X^{0}$ is unstable.

\subsection{Sensitivity analysis}

It is important to know the relative importance of some factors that maintain or not a mirid population. We may estimate the sensitivity index of $\mathcal{N}_{0}$ with respect to a parameter $p$, as follows:

$$
\gamma_{p}^{\mathcal{N}_{0}}=\frac{\partial \mathcal{N}_{0}}{\partial p} \cdot \frac{p}{\mathcal{N}_{0}}
$$

Straightforward calculation leads to the following result:

$$
\begin{aligned}
& \gamma_{r}^{\mathcal{N}_{0}}=\gamma_{b}^{\mathcal{N}_{0}}=1, \quad \gamma_{\nu_{E}}^{\mathcal{N}_{0}}=\frac{\mu_{E}}{\nu_{E}\left(\nu_{E}+\mu_{E}\right)}, \\
& \gamma_{\nu_{L}}^{\mathcal{N}_{0}}=\frac{\mu_{L}}{\nu_{L}\left(\nu_{L}+\mu_{L}\right)}, \quad \gamma_{\nu_{F}}^{\mathcal{N}_{0}}=\frac{\mu_{F}}{\nu_{F}\left(\nu_{F}+\mu_{A}\right)}, \\
& \gamma_{\mu_{E}}^{\mathcal{N}_{0}}=-\frac{1}{\nu_{E}+\mu_{E}}, \quad \gamma_{\mu_{L}}^{\mathcal{N}_{0}}=-\frac{1}{\nu_{L}+\mu_{L}}, \quad \gamma_{\mu_{A}}^{\mathcal{N}_{0}}=-\frac{\nu_{F}}{\mu_{A}\left(\nu_{F}+\mu_{A}\right)}
\end{aligned}
$$

Clearly, $r$ and $b$ have the strongest impact on $\mathcal{N}_{0}$. However, this gives us only partial informations. In particular, we will now focus on the variables $E, L, F_{1}, F_{2}$ and $M$. That is why, we derive some global sensitivity analysis using two well-known methods: the eFast and the LHS-PRCC methods. The eFast method given in Fig.2, page 9, highlights first-order effects (main effects) and total effects (main and all interaction effects) of the parameters on the Model Outputs. Complementary to the eFast method, we derived a LHS-PRCC sensitivity analysis given in Fig.3, page 10. LHSPRCC stands for Latin Hypercube Sampling and PRCC for Partial rank correlation coefficient. These two methods give complementary information. Indeed the PRCC method provides mainly information about how the outputs is impacted if we increase (or decrease) the inputs of a specific parameter while the eFast indicates which parameter uncertainty has the greatest impact on the output variability (see for instance [36] for further explanations).

In Table 2, page 8 , we provide ranges of values for the model parameters. These ranges were estimated based on the Data obtained by Babin and collaborators. 


\begin{tabular}{|c|l|l|}
\hline Parameters & Range & Source \\
\hline$r$ & {$[0.5,0.6]$} & estimated \\
\hline$b$ & {$[1.5 ; 4]$} & estimated \\
\hline$K_{C}$ & {$[1,10000]$} & estimated \\
\hline$\nu_{L}$ & {$[1 / 35,1 / 10]$} & estimated \\
\hline$\nu_{F}$ & {$[1 / 10,1 / 6]$} & estimated \\
\hline$\mu_{L}$ & {$[1 / 100,1 / 10]$} & estimated \\
\hline$\mu_{A}$ & {$[1 / 100,1 / 10]$} & estimated \\
\hline$\mu_{E}$ & {$[1 / 100,1 / 10]$} & estimated \\
\hline$\nu_{E}$ & {$[1 / 10,1 / 20]$} & estimated \\
\hline
\end{tabular}

Table 2: Range of values for the parameters of system (1).

As expected, variation of the carrying capacity $K_{C}$ has strong an impact on all variables, and also $\mu_{A}$. For the transition rates $\nu_{L}, \nu_{E}, \nu_{F}$, and the mortality rate $\mu_{L}$, their impact is weaker, but according to the LHS-PRCC analysis, is not negligible. Thus obviously, among all parameters, the parameters $K_{C}, \mu_{A}, \nu_{L}, \nu_{E}, \nu_{F}$, and $\mu_{L}$ are the most important parameters to estimate. Finally, according to the sensitivity analysis method that is considered $b$ does not have the same impact. However, we think that this is an important parameter too.

\subsection{Mirid system with periodic coefficients}

In this section, we consider the previous model, but with periodic time-dependent parameters, since we know that most of the parameters may depend on the environment. In fact, mirids dynamics vary greatly during the year; density of populations is likely to be influenced by pods avail ability on the trees. In [12], it is admitted that lower mirid populations observed in the plots during a certain period of the year is due to the declining fertility of females and increasing mortality of individuals. Thus it seems that development parameters (longevity, fecundity, mortality) of mirids vary depending on season. We assume that all those parameters are $T$-periodic functions and are bounded: below, by a non negative minimal value, and above by a positive maximal value, that is $p_{\min } \leq p(t) \leq p_{\max }$ for all $t \geq 0$, and $p=r, b, \mu_{L}, \mu_{A}, \mu_{E}, \nu_{L}, \nu_{F}, \nu_{E}$ or $K_{C}$. We also split the carrying capacity in two parts, $K_{C}(t)+C: K_{C}(t)$, the mean number of pods available for breeding, and $C$, the alternative breeding sites including other tree hosts, like Cola nitida, Ceiba pentandra [11]. Thus, Model (1) becomes

$$
\left\{\begin{array}{l}
\dot{E}=b(t) F_{2}\left(1-\frac{E}{C+K_{C}(t)}\right)-\left(\nu_{E}(t)+\mu_{E}(t)\right) E, \\
\dot{L}=\nu_{E}(t) E-\left(\nu_{L}(t)+\mu_{L}(t)\right) L, \\
\dot{F}_{1}=r(t) \nu_{L}(t) L-\left(\nu_{F}(t)+\mu_{A}(t)\right) F_{1}, \\
\dot{F}_{2}=\nu_{F}(t) F_{1}-\mu_{A}(t) F_{2}, \\
E(0)=E^{0}, \quad L(0)=L^{0}, \quad F_{1}(0)=F_{1}^{0}, \quad F_{2}(0)=F_{2}^{0}
\end{array}\right.
$$

In fact system (8) enters the family of periodic concave cooperative system with a concave nonlinearity $([30],[43])$.

Definition 2.1 Let us consider a n-dimensional autonomous differential system:

$$
\dot{x}=F(t, x), \quad x \in \mathbb{R}^{n}
$$



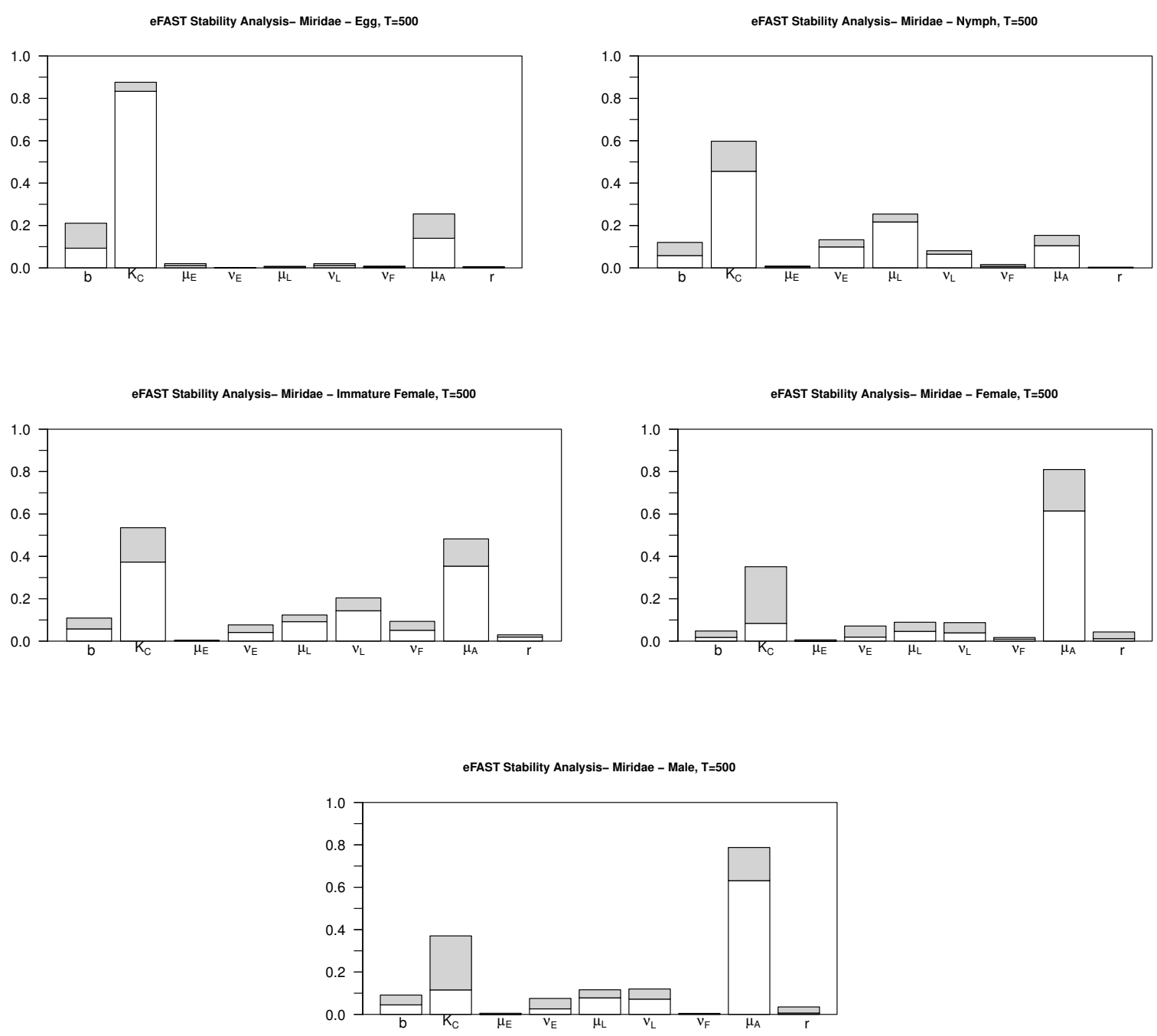

Figure 2: e-FAST Sensitivity analysis at $T=500$. White bar:first-order effects; Sum of white and grey bars: total effect.

The system of differential equations (9) is called cooperative with concave non-linearities if

(i) $\frac{\partial F_{i}}{\partial x_{j}} \geq 0, i \neq j$ for each $(t, x) \in \mathbb{R}_{+} \times \mathbb{R}_{+}^{n}$.

(ii) $x>y>0$ implies $D_{x} F(t, y)>D_{x} F(t, x)$.

We will use a theorem (Theorem 3.1, see appendix B) proved in [43]. We have:

$$
F(t, x)=\left(\begin{array}{c}
b(t) F_{2}\left(1-\frac{E}{C+K_{C}(t)}\right)-\left(\nu_{E}(t)+\mu_{E}(t)\right) E \\
\nu_{E}(t) E-\left(\nu_{L}(t)+\mu_{L}(t)\right) L \\
r(t) \nu_{L}(t) L-\left(\nu_{F}(t)+\mu_{A}(t)\right) F_{1} \\
\nu_{F}(t) F_{1}-\mu_{A}(t) F_{2}
\end{array}\right),
$$



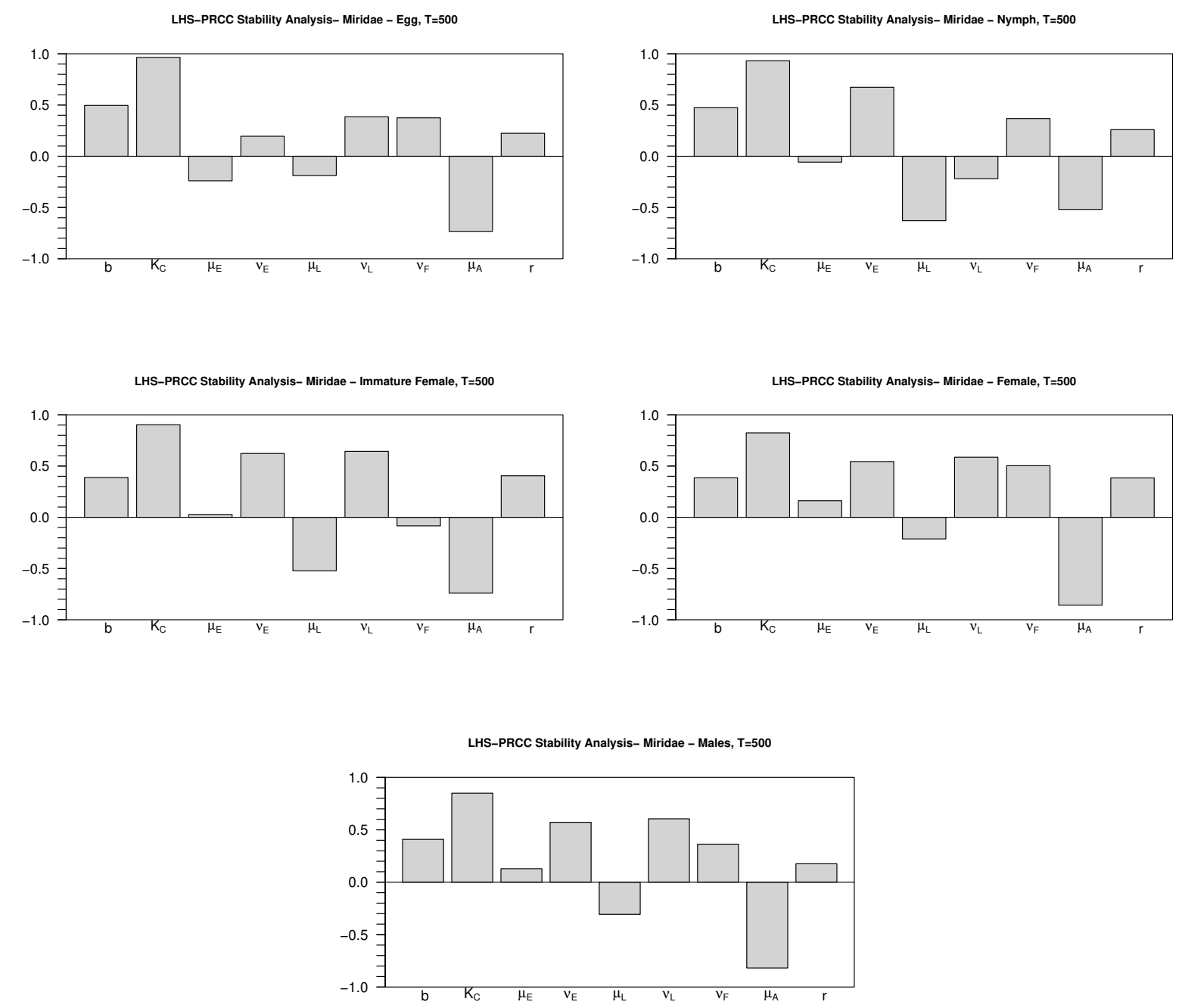

Figure 3: LHS-PRCC Sensitivity analysis at $T=500$.

where $x=\left(E, L, F_{1}, F_{2}\right)^{T}$. Obviously, $F$ is continuously differentiable and $T$-periodic. According to Cauchy-Lipschitz theorem, we have existence of a positive and bounded solution. Since all timedependent parameters are positive, we verify easily property (i) in Definition 2.1.

Let us compute the Jacobian

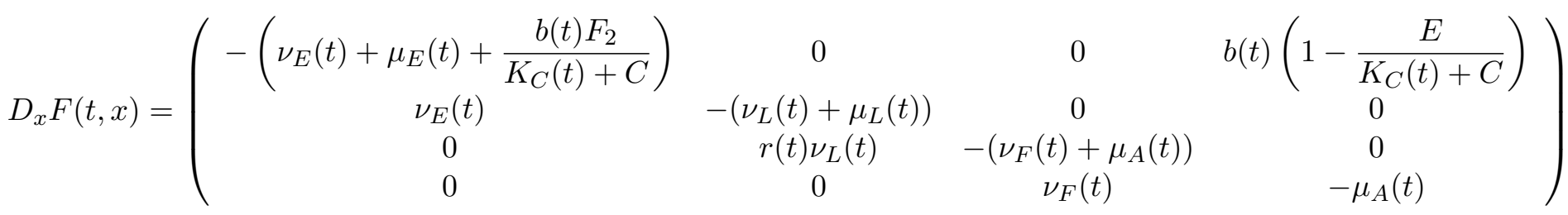

It is straightforward to verify (ii) in Definition 2.1. In addition, $D_{x} F(t, x)$ is irreducible for each $(t, x) \in \mathbb{R} \times \mathbb{R}_{+}^{n}$. Thus, Theorem .1 applies to system (9) and we deduce that every solution $x$, with $x\left(t_{0}\right) \geq 0$, can be continued in $\left[t_{0}, \infty\right]$ with $x(t) \geq 0$ for $t \geq t_{0}$. 
Now, let us compute $D_{x} F(t, 0)$, that is

$$
D_{x} F(t, 0)=\left(\begin{array}{cccc}
-\left(\nu_{E}(t)+\mu_{E}(t)\right) & 0 & 0 & b(t) \\
\nu_{E}(t) & -\left(\nu_{L}(t)+\mu_{L}(t)\right. & 0 & 0 \\
0 & r(t) \nu_{L}(t) & -\left(\nu_{F}(t)+\mu_{A}(t)\right) & 0 \\
0 & 0 & \nu_{F}(t) & -\mu_{A}(t)
\end{array}\right)
$$

According to Theorem .1, if the Floquet multipliers of $D_{x} F(t, 0)$ lie inside or on the unit circle, then $\lim _{t \rightarrow \infty} x(t)=0$. In contrary, if any Floquet multiplier lies outside the unit circle, and according to the fact that $F(t, 0) \equiv 0$ and $x$ is a bounded solution, we deduce that system (9) has a unique non-zero $T$-periodic solution $x_{p e r}(t)$.

In general, the determination of Floquet multipliers is extremely difficult. That is why, we will now consider an additional result [30] recalled in Appendix B (see Theorem .2). This is an algebraic criterion, related to the study of $A(t)=D_{x} F(t, 0)$.

Let us estimate $\underline{\mathrm{A}}$ and $\bar{A}$, lower and upper bounds of Matrix $A(t)$. Using the fact that all timedependent parameters have positive lower and upper bounds, we deduce

$$
\underline{\mathrm{A}}=\left(\begin{array}{cccc}
-\left(\nu_{E, \max }+\mu_{E, \max }\right) & 0 & 0 & b_{\min } \\
\nu_{E, \min } & -\left(\nu_{L, \max }+\mu_{L, \max }\right) & 0 & 0 \\
0 & r_{\min } \nu_{L, \min } & -\left(\nu_{F, \max }+\mu_{A, \max }\right) & 0 \\
0 & 0 & \nu_{F, \min } & -\mu_{A, \max }
\end{array}\right)
$$

and

$$
\bar{A}=\left(\begin{array}{cccc}
-\left(\nu_{E, \min }+\mu_{E, \min }\right) & 0 & 0 & b_{\max } \\
\nu_{E, \max } & \left(\nu_{E, \min }+\mu_{E, \min }\right) & 0 & 0 \\
0 & r_{\max } \nu_{L, \max } & -\left(\nu_{F, \min }+\mu_{A, \min }\right) & 0 \\
0 & 0 & \nu_{F, \max } & -\mu_{A, \min }
\end{array}\right) .
$$

Then according to Theorem .2, we have to study the principal minors of $-\underline{\mathrm{A}}$ and $-\bar{A}$. All diagonal terms of $-\underline{\mathrm{A}}$ and $-\bar{A}$ are positive. In fact it suffices to compute $\operatorname{det}(-\bar{A})$ and $\operatorname{det}(-\underline{\mathrm{A}})$, that is

$\operatorname{det}(-\bar{A})=-\left(\nu_{E, \min }+\mu_{E, \min }\right)\left(\nu_{L, \min }+\mu_{L, \min }\right)\left(\nu_{F, \min }+\mu_{A, \min }\right) \mu_{A, \min }+b_{\max } r_{\max } \nu_{E, \max } \nu_{L, \max } \nu_{F, \max }$,

$\operatorname{det}(-\underline{\mathrm{A}})=\left(\nu_{E, \max }+\mu_{E, \max }\right)\left(\nu_{L, \max }+\mu_{L, \max }\right)\left(\nu_{F, \max }+\mu_{A, \max }\right) \mu_{A, \max }-b_{\min } r_{\min } \nu_{L, \min } \nu_{F, \min }$.

Thus $\operatorname{det}(-\bar{A}) \geq 0$ if

$$
b_{\max } r_{\max } \nu_{L, \max } \nu_{F, \max } \geq\left(\nu_{E, \min }+\mu_{E, \min }\right)\left(\nu_{L, \min }+\mu_{L, \min }\right)\left(\nu_{F, \min }+\mu_{A, \min }\right) \mu_{A, \min },
$$

and $\operatorname{det}(-\underline{\mathrm{A}})<0$ if

$$
\left(\nu_{E, \max }+\mu_{E, \max }\right)\left(\nu_{L, \max }+\mu_{L, \max }\right)\left(\nu_{F, \max }+\mu_{A, \max }\right) \mu_{A, \max }<b_{\min } r_{\min } \nu_{L, \min } \nu_{F, \min } .
$$

In fact, the previous results are related to the time dependent basic offspring number

$$
\mathcal{N}_{0}(t)=\frac{r b(t) \nu_{E}(t) \nu_{L}(t) \nu_{F}(t)}{\mu_{A}(t)\left(\nu_{E}(t)+\mu_{E}(t)\right)\left(\nu_{F}(t)+\mu_{A}(t)\right)\left(\nu_{L}(t)+\mu_{L}(t)\right)},
$$

such that $\mathcal{N}_{0, \text { min }} \leq \mathcal{N}_{0}(t) \leq \mathcal{N}_{0, \text { max }}$, where

$$
\mathcal{N}_{0, \text { min }}=\frac{r_{\min } b_{\min } \nu_{E, \min } \nu_{L, \min } \nu_{F, \min }}{\left(\nu_{E, \max }+\mu_{E, \max }\right)\left(\nu_{\max }+\mu_{\max }\right)\left(\nu_{F, \max }+\mu_{A, \max }\right) \mu_{A, \max }},
$$


and

$$
\mathcal{N}_{0, \text { max }}=\frac{r_{\max } b_{\max } \nu_{E, \max } \nu_{L, \max } \nu_{F, \max }}{\left(\nu_{E, \min }+\mu_{E, \min }\right)\left(\nu_{\min }+\mu_{\min }\right)\left(\nu_{F, \min }+\mu_{A, \min }\right) \mu_{A, \min }} .
$$

According to Theorem .2, we deduce

\section{Theorem 2.3 :}

(i) If $\mathcal{N}_{0, \text { max }} \leq 1$, then the solution of system (8) converges to the trivial equilibrium $X^{0}$.

(ii) If $\mathcal{N}_{0, \text { min }}>1$, then system (8) admits a unique periodic solution that attracts all initial condition in $\Omega$.

According to the sensitivity analysis, an interesting and particular case is when we assume that all parameters are constant, except $K$. Then $\bar{A}=\underline{A}$, and thus $\mathcal{N}_{0, \max }=\mathcal{N}_{0, \min }=\mathcal{N}_{0}$. Thus, theorem 2.3 reduces to

Theorem 2.4 (i) If $\mathcal{N}_{0} \leq 1$, then the solution of system (8) converges to the trivial equilibrium $X^{0}$.

(ii) If $\mathcal{N}_{0}>1$, then system (8) admits a unique periodic solution which attracts all initial condition in $\Omega$.

In fact, we recover the same results than for the constant parameters problem, except that the constant positive equilibrium is now periodic of period $T$.

It is important to notice that $K_{C}$ constant is relevant in the case where the cocoa production is almost constant along the year. This is realistic in Central America where there is no real seasonality. By contrast, in Cameroon, they are two rainy season: a long one, and a short one. There the seasonality is clearly marked, which has an impact on cacao production. That is why we consider a periodic function for $K_{C}$.

\section{Numerical simulation}

Now, we will illustrate our theoretical results. We will use the values of the monthly mean number of pods using data extrapolated, obtained in Cameroon, from [16] to construct our periodicfunction $K_{C}(t)$. According to the data from [16] and the knowledge about the mean number of pods over the year, our daily estimated data are recapitulated in Table 3 . We suppose that the

\begin{tabular}{|c|c|c|c|c|c|c|c|c|c|c|c|c|c|}
\hline Months & Jun & Jul & Aug & Sept & Oct & Nov & Dec & Jan & Feb & Mar & Apr & May & Jun \\
\hline$K_{C}(t)$ & 0 & $\frac{32000}{31}$ & $\frac{160000}{31}$ & $\frac{416000}{30}$ & $\frac{544000}{31}$ & $\frac{304000}{31}$ & $\frac{416000}{31}$ & $\frac{120000}{31}$ & $\frac{8000}{28}$ & 0 & 0 & 0 & 0 \\
\hline
\end{tabular}

Table 3: Mean number of pods per days

function $K_{C}(t)$ is periodic, of period $T=365$ days. We use the cubic spline interpolation to derive the time evolution of $K_{C}(t)$ along a year. Using cubic Hermite spline, we obtain a polynomial interpolation (Daily estimation of $K_{C}(t)$ ) given in Fig. 4, page 13. Figure 4, page 13 represents the daily estimation of pods number in the plot. $K_{C}(t)$.

When the parameter $K_{C}$ is constant, we used a classical scheme already well implemented under matlab (ode23s) for numerical simulations and we consider that the number of pods $K_{C}$ is constant, we evaluate the average daily value estimated with the data of Table 3 , that is

$$
\bar{K}_{C}=\frac{1}{365} \int_{1}^{365} K_{C}(t) d t
$$




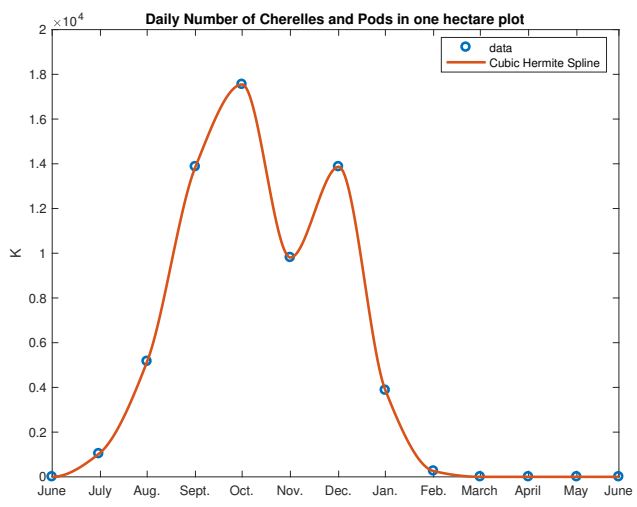

Figure 4: Daily estimation of $K_{C}(t)$ along a year

- When $K_{C}$ is periodic, following [6], we consider a nonstandard numerical scheme to preserve most of the qualitative properties of the continuous model, like positivity, equilibria, stability and instability whatever the time step $\Delta t>0$. We consider the values of parameters given in Table 4 . We illustrate our numerical simulations for two values of $C: C=5$ and $C=100$. All the given values have been estimated thanks to $[7,31,48,13,46]$.

\begin{tabular}{|c|c|c|c|}
\hline Parameters & Case $\mathcal{N}_{0}<1$ & Case $\mathcal{N}_{0}>1$ & Source \\
\hline$r$ & 0.58 & 0.58 & {$[13]$} \\
\hline$b$ & 3.28 & 3.28 & Estimated \\
\hline$\nu_{E}$ & $1 / 15$ & $1 / 15$ & Estimated \\
\hline$\nu_{L}$ & $1 / 25$ & $1 / 25$ & Estimated \\
\hline$\nu_{F}$ & $1 / 10$ & $1 / 10$ & Estimated \\
\hline$\mu_{E}$ & 0.05 & 0.001 & Estimated \\
\hline$\mu_{L}$ & 0.15 & 0.03 & Estimated \\
\hline$\mu_{A}$ & 0.15 & 0.07 & Estimated \\
\hline \hline $\mathcal{N}_{0}$ & 0.6103 & 9.0002 & Equation (4) \\
\hline
\end{tabular}

Table 4: Values of constant parameters

In Fig. 5, page 14, we illustrate the theoretical results when the carrying capacity $K_{C}$ is constant, that is $\bar{K}_{C}=5482$, with initial conditions $\left(E, L, F_{1}, F_{2}, M\right)=(0,0,0,10,10)$. As expected, when $\mathcal{N}_{0}<1$, the population decays till extinction, while, when $\mathcal{N}_{0}>1$, the population reaches rapidly the positive equilibrium. However, in Cameroon, having a constant number of pods along the year is not realistic. That's why in the next simulations, we consider a more realistic case, with the same parameters values. In Figs. 6 and 7, page 14, we illustrate the previous results when the carrying capacity $K_{C}$ is periodic. However, in the case where $\mathcal{N}_{0}>1$, the dynamic of mirids follows the dynamic of pods, as expected. However, the size of the population seems to be large compared to real observations (see for instance [16]).

\section{A model with delays}

In the previous section, we studied the time evolution of mirid population as if the transition 


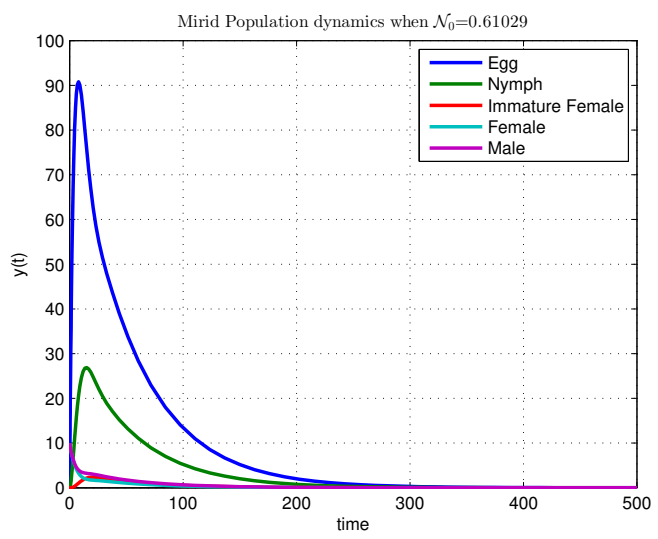

(a)

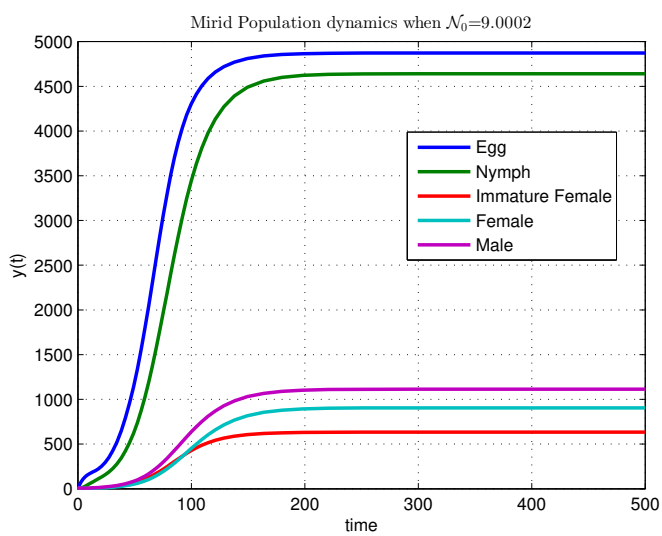

Figure 5: Time Evolution of mirid population when $K_{C}=5482 ;(\mathrm{a}) \mathcal{N}_{0}<1 ; \quad$ (b) $\mathcal{N}_{0}>1$.
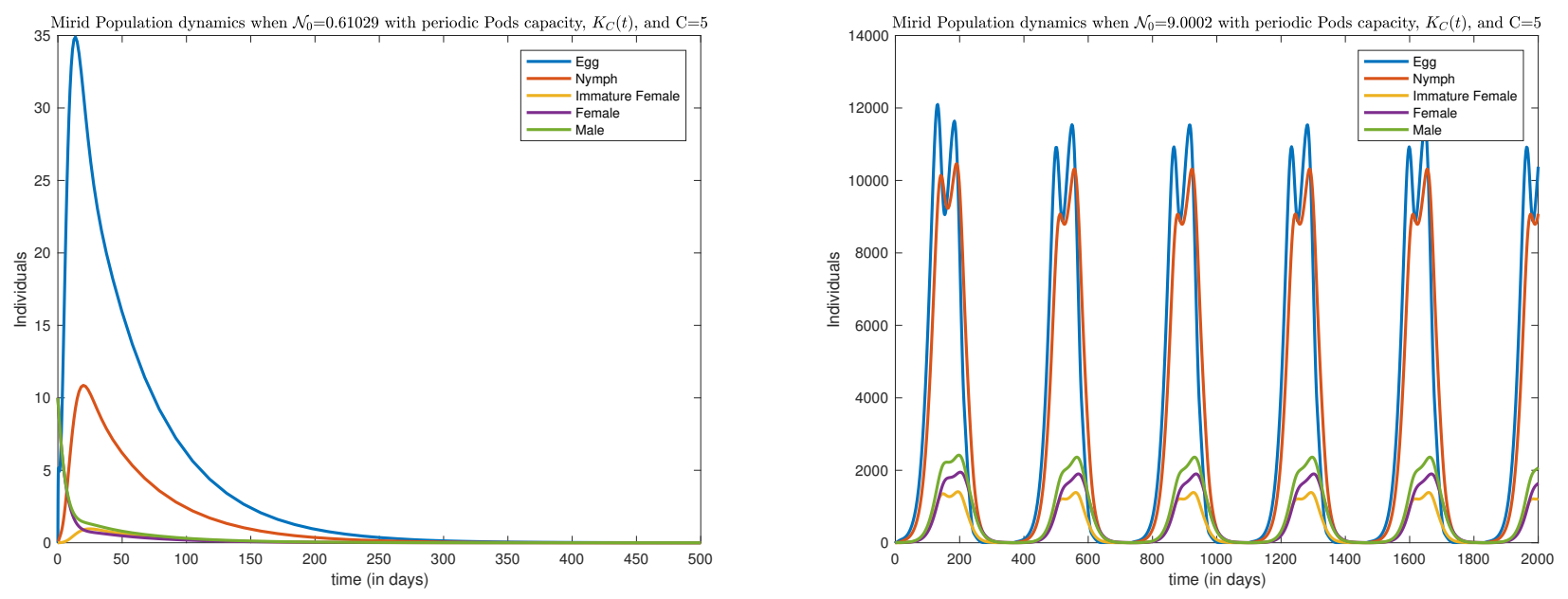

Figure 6: Time Evolution of mirid population when $K_{C}$ is periodic and $C=5 ; \quad$ (a) $\mathcal{N}_{0}<1$; $\mathcal{N}_{0}>1$.

to one stage of development to another is immediate. Biologically, this is not correct: let us return on the biological life cycle of $S$. singularis given in Fig. 8, page 15. In fact, each individual needs to stay a certain amount of time in each compartment to complete its stage, in particular in the egg, nymph and non-mature female stages: there exists $\tau_{1}$ days between egg-laying and appearing of new nymphs; $\tau_{2}$ days between nymphs and emergence to adults. In addition, female needs $\tau_{3}$ days to become mature before being able to lay eggs. In this section, we will take into account some of these previous times leading to time-delayed model with delays.

Now, it is possible to take into account different biological facts. In particular, based on the literature, we know that after deposition, eggs need (in mean) $\tau_{1}=15$ days to hatch and enter the nymphs compartment. $b$ always represents the mean daily number of eggs laid by an adult female. $\tau_{2}=25$ days represent the required time for nymphs to achieve their development and become adults. So only a proportion $e^{-\tau_{2} \mu_{L}}$ of Nymphs will survive and become adults, . Thus $\nu_{E} e^{-\tau_{2} \mu_{L}} E\left(t-\tau_{2}\right)$ represents the transition rate from eggs to adults, after $\tau_{2}$ days in the Nymphs compartment. The term $r e^{-\tau_{3} \mu_{A}}$ represents the proportion of immature adults that will deposit 

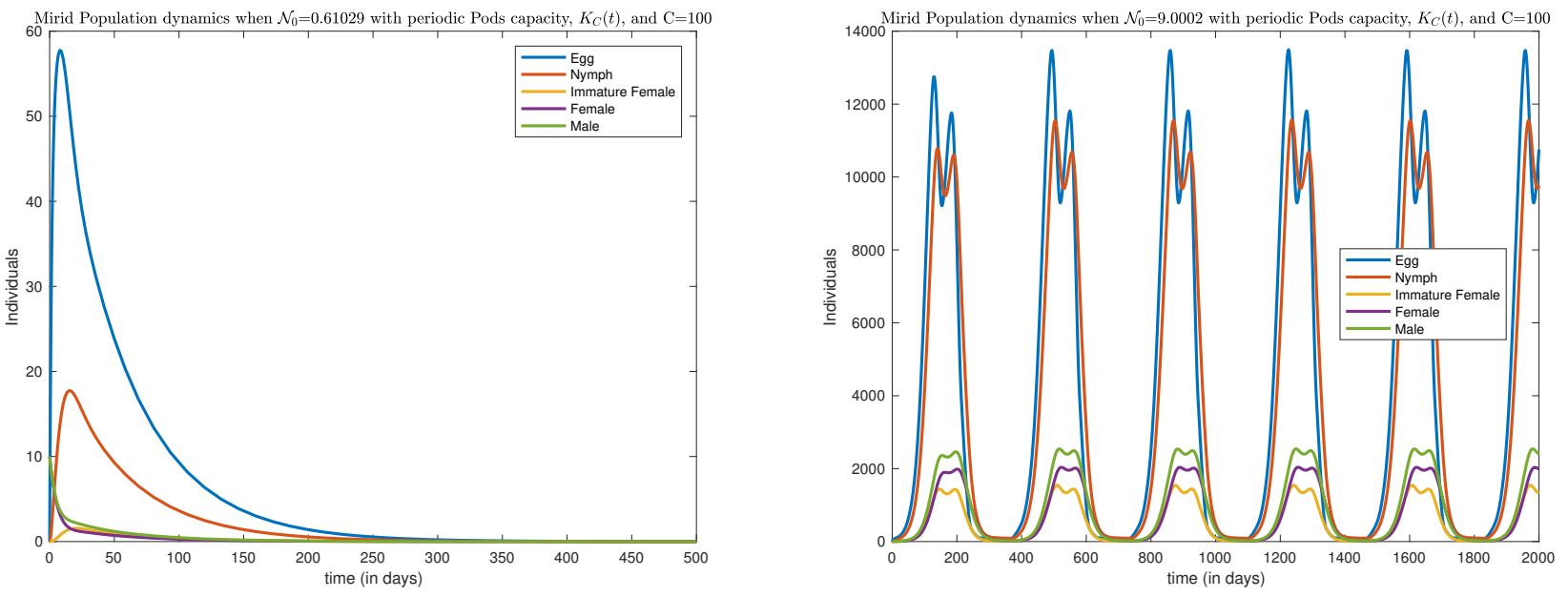

Figure 7: Time Evolution of mirid population when $K_{C}$ is periodic and $C=100$; (b) $\mathcal{N}_{0}>1$.

(a) $\mathcal{N}_{0}<1$

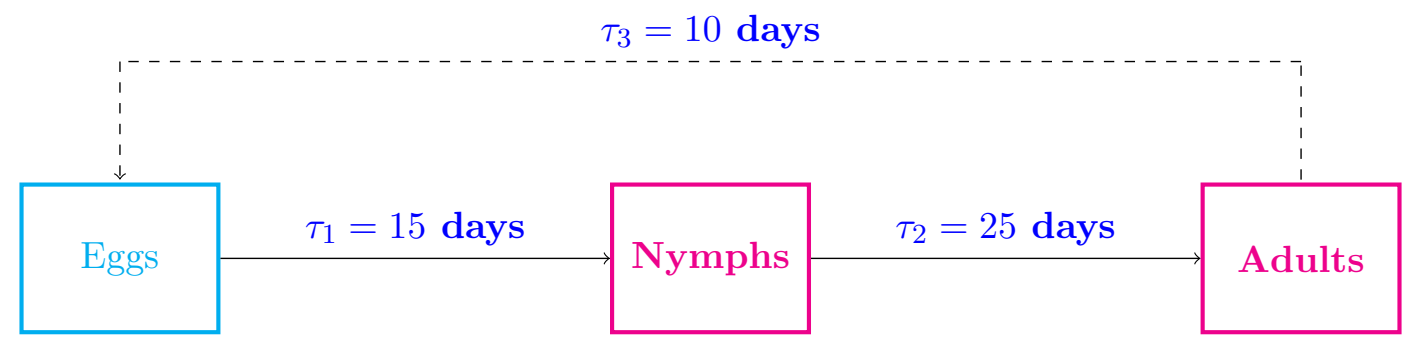

Figure 8: Life cycle of $S$. singularis

eggs, after $\tau_{3}$ of maturation.

Altogether, we obtain the following "two-delays" model:

$$
\left\{\begin{array}{l}
\frac{d E}{d t}=r b e^{-\tau_{3} \mu_{A}} A\left(t-\tau_{3}\right)\left(1-\frac{E}{K_{C}}\right)-\left(\nu_{E}+\mu_{E}\right) E \\
\frac{d A}{d t}=\nu_{E} e^{-\tau_{2} \mu_{L}} E\left(t-\tau_{2}\right)-\mu_{A} A
\end{array}\right.
$$

$A=F_{1}+F_{2}+M$ and this is why the sex ratio $r$ enters the $E$ equation in Eq. (10). System (10) can be rewritten as follows:

$$
\left\{\begin{array}{l}
\frac{d x_{1}}{d t}=\alpha x_{2}\left(t-\tau_{3}\right)\left(1-\frac{x_{1}}{K_{C}}\right)-\beta x_{1} \\
\frac{d x_{2}}{d t}=\gamma x_{1}\left(t-\tau_{2}\right)-\delta x_{2}
\end{array}\right.
$$

where

$$
\alpha=r b e^{-\tau_{3} \mu_{A}}, \quad \beta=\nu_{E}+\mu_{E}, \quad \gamma=\nu_{E} e^{-\tau_{2} \mu_{L}}, \quad \delta=\mu_{A} .
$$

The right-hand side of system (11) is continuous and Lipschitzian in $x$. Thus, according to the standard theory of Delay Differential Equations [29], system (11) admits a unique solution for each continuous initial condition $\varphi \in \mathcal{C}\left([-\tau ; 0], \mathbb{R}^{2}\right)$ where $\tau=\max \left(\tau_{3}, \tau_{2}\right)$. We denote $\mathcal{C}\left([-\tau ; 0], \mathbb{R}^{2}\right)$ the 
Banach space of continuous functions mapping the interval $[-\tau ; 0]$ into $\mathbb{R}^{2}$ with the topology of uniform convergence; i.e. for $\varphi \in \mathcal{C}\left([-\tau ; 0], \mathbb{R}^{2}\right)$, the norm of $\varphi$ is defined as $\|\varphi\|=\sup _{-\tau \leq \theta \leq 0}|\varphi(\theta)|$ where $|$.$| is a norm of \mathbb{R}^{2}$.

Let

$$
\mathcal{D}=\left\{x \in \mathbb{R}_{+}^{2} / x_{1} \leq K_{C}, \quad x_{2} \leq \frac{\gamma K_{C}}{\delta}\right\}
$$

System (11) can be rewritten as follows

$$
x^{\prime}(t)=f\left(x(t), x\left(t-\tau_{3}\right), x\left(t-\tau_{2}\right)\right)=f(x, Y),
$$

where $Y=\left(x\left(t-\tau_{3}\right), x\left(t-\tau_{2}\right)\right)$. Following [44], it is important to notice that system (8) without delays reduces to a cooperative irreducible system. In fact the delayed system is cooperative too. Indeed, according to $[44,42]$, we can show that $f$ verifies the quasimonotone condition, defined as follows in $[44,42]$

$$
\phi, \psi \in \mathcal{D}, \phi \leq \psi \text { and } \phi_{i}(0)=\psi_{i}(0) \quad \text { implies } \quad f_{i}(\phi) \leq f_{i}(\psi) .
$$

In fact, it suffices to use Theorem 4.5 [42], page 308, i.e. to show that $f$ is cooperative to deduce that (13) holds for $f$, that is

$$
\begin{aligned}
& \frac{\partial f_{i}}{\partial x_{j}}(x, Y) \geq 0, \quad \text { for } i \neq j, \\
& \frac{\partial f_{i}}{\partial y_{j}^{k}}(x, Y) \geq 0, \quad \text { for all } i, j, k .
\end{aligned}
$$

Obviously, since the non-delayed model is cooperative, condition (14) is verified. Let us now check condition (15):

$$
\begin{aligned}
& \frac{\partial f_{1}}{\partial y_{1}^{1}}(x, Y)=0, \quad \frac{\partial f_{1}}{\partial y_{2}^{1}}(x, Y)=\alpha\left(1-\frac{x_{1}}{K_{C}}\right) \geq 0, \\
& \frac{\partial f_{2}}{\partial y_{1}^{2}}(x, Y)=\gamma>0, \quad \frac{\partial f_{2}}{\partial y_{2}^{2}}(x, Y)=0 .
\end{aligned}
$$

Since the previous conditions are verified we deduce that $f$ is quasi-monotone, which implies that if the initial condition is positive (with at most one zero component), then the solution $x$ is still non-negative, i.e. $x(t) \geq 0$.

Similarly, using the fact that the initial condition $\phi \in \mathcal{D}$, we have $x_{1} \leq K_{C}$, and $x_{2} \leq \frac{\gamma K_{C}}{\delta}$, for $t \in[-\tau, 0]$. Using these inequalities in (11), we infer that this is still true when $t \in[0, \tau]$. Iterating this reasoning, we finally deduce that $x(t) \in \mathcal{D}$ for all $t \geq 0$.

A direct computation shows that system (11) admits two equilibria: the trivial ones, $\mathbf{0}=(0,0)$, and

$$
\mathbf{x}^{*}=\left(\left(1-\frac{\beta \delta}{\alpha \gamma}\right) K_{C}, \frac{\gamma}{\delta}\left(1-\frac{\beta \delta}{\alpha \gamma}\right) K_{C}\right)=\left(\left(1-\frac{1}{\mathcal{R}}\right) K_{C}, \frac{\gamma}{\delta}\left(1-\frac{1}{\mathcal{R}}\right) K_{C}\right),
$$

when $\mathcal{R}>1$ where

$$
\mathcal{R}=\frac{\alpha \gamma}{\beta \delta}=\frac{r b \nu_{E} e^{-\tau_{2} \mu_{L}-\tau_{3} \mu_{A}}}{\mu_{A}\left(\nu_{E}+\mu_{E}\right)}
$$

Note that

$$
\mathcal{R}=\frac{\alpha \gamma}{\beta \delta}=\frac{r b \nu_{E} e^{-\tau_{2} \mu_{E}-\tau_{3} \mu_{A}}}{\mu_{A}\left(\nu_{E}+\mu_{E}\right)}<\mathcal{N}=\frac{r b \nu_{E} \nu_{L} \nu_{F}}{\mu_{A}\left(\nu_{E}+\mu_{E}\right)\left(\nu_{L}+\mu_{L}\right)\left(\nu_{F}+\mu_{A}\right)}
$$


Thus, for some parameters values, we may have $\mathcal{N}>1$ and $\mathcal{R}<1$.

There is no need to study the stability/instability of the equilibria. Indeed, according to [44] (Chapter 5), there is a nice property pointed out about cooperative irreducible time-delay systems: the asymptotic stability of each equilibrium is preserved for the delay differential system (8), whatever the values taken by the delays. In particular, when $\mathcal{R} \leq 1(\mathcal{R}>1)$, all orbits are attracted by $\mathbf{0}\left(\mathbf{x}^{*}\right)$. In other words, to study the long term behaviour of cooperative time-delay systems it suffices to study the cooperative systems without delay(s).

This result implies that the use of delays does not change the long-time behaviour of the timedependent system (8). However, when the system is non-autonomous and periodic, its behaviour may be different in the transient period from the non-delayed non-autonomous periodic system as it is showed in the forthcoming simulations (see Fig. 11, page 21), and Fig.12, page 21).

Remark 3.1 The previous time-delayed system is considered with fixed delays. A possible extension, for future work would be to consider time varying delays, $\tau_{i}(t)$, since it seems obvious to consider that the developmental time in each stage may change according to environmental parameters, like temperature, rainfall.... Finally, distributed delays could be considered. Unfortunately for both cases, we do not have data.

Finally, like for the non-delayed model, it is interesting to provide a global sensitivity analysis at different time $T=100$ and $T=500$ : see Fig. 9, page 18. Clearly, the parameters $\mu_{L}$, and $\mu_{A}$ are the most sensitive parameters, and the delays play mainly a role in the transient phase $(T=100)$ and no more at equilibrium, contrary to the carrying capacity, $K_{C}$, even if this parameter is far less sensitive than in the previous non-delayed model. This clearly shows the importance of estimating efficiently these parameters in different environmental or semi-field conditions. It also very interesting to compare the sensitivity analysis between two models that are supposed to model the same system.

\section{Numerical simulation}

Now, we illustrate all the different stability cases, i.e. when $\mathcal{R}<1$ and $\mathcal{R}>1$. The timedelayed Model is solved using Matlab and the dde23 function. In Table 4, page 13 we summarize the parameters values used in the next simulations but we attribute the new values of parameter $\mu_{L}$. Then, the values of parameters for those simulations are consigned in Table 5, page 19 .

\begin{tabular}{|c|c|c|c|}
\hline Parameters & Case $\mathcal{R}<1$ & Case $\mathcal{R}>1$ & Source \\
\hline$b$ & 3.28 & 3.28 & Estimated \\
\hline$\tau_{2}$ & 25 & 25 & Estimated \\
\hline$\tau_{3}$ & 10 & 10 & Estimated \\
\hline$\mu_{A}$ & 0.1 & 0.07 & Estimated \\
\hline $\mathrm{r}$ & 0.58 & 0.58 & {$[13]$} \\
\hline$\mu_{L}$ & 0.1 & 0.03 & Estimated \\
\hline$\nu_{E}$ & $1 / 15$ & $1 / 15$ & Estimated \\
\hline$\mu_{E}$ & 0.001 & 0.001 & Estimated \\
\hline \hline $\mathcal{R}$ & 0.566 & 6.28 & Equation (18) \\
\hline
\end{tabular}

Table 5: Values of constant parameters for the time-delay model 

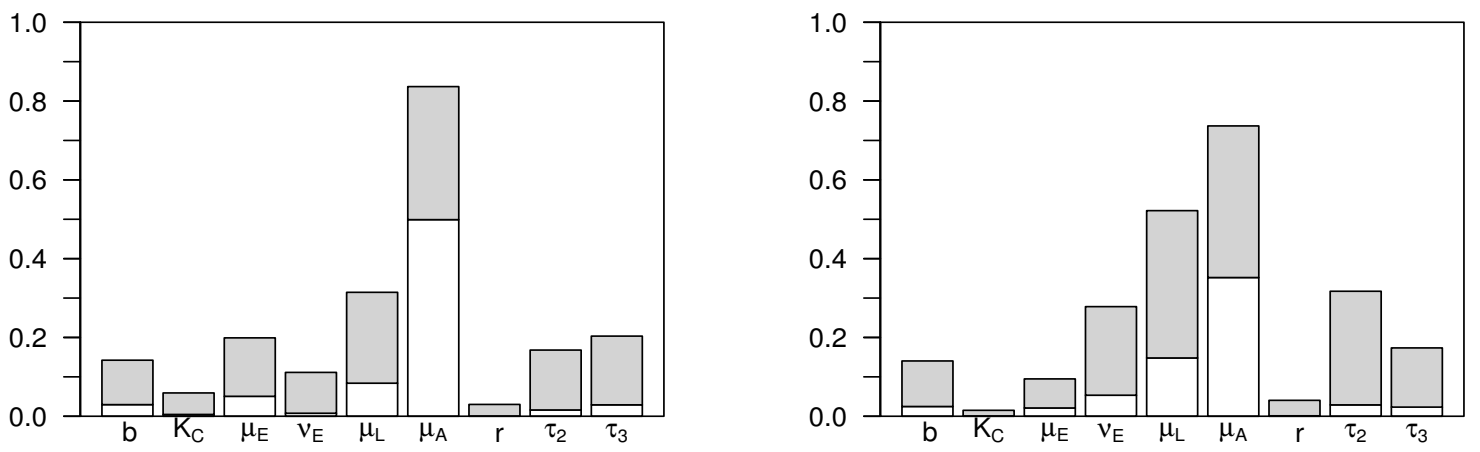

eFAST Stability Analysis- Delayed Model - Egg, T=500
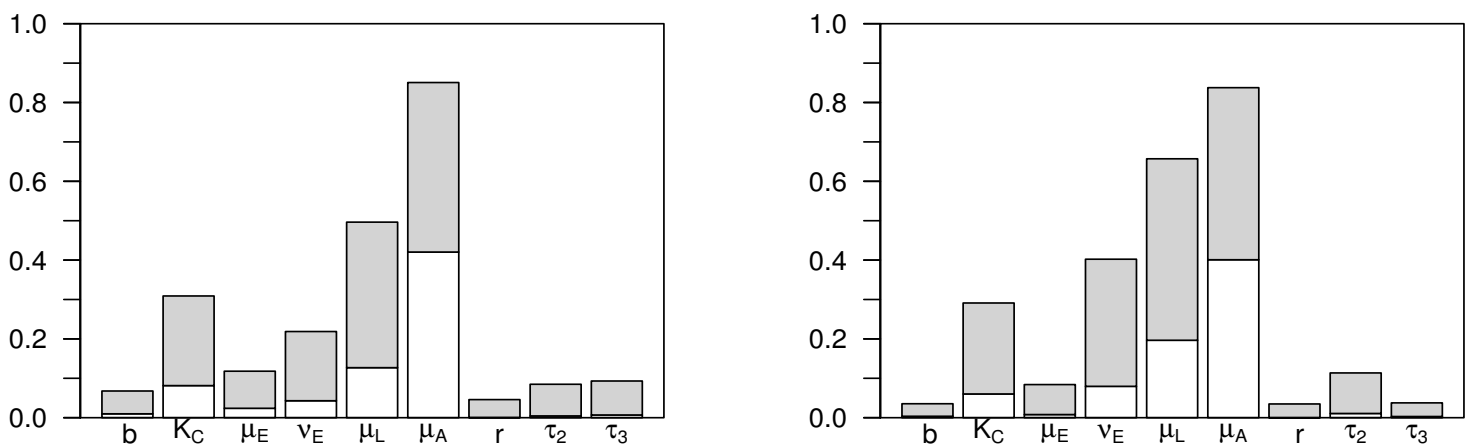

Figure 9: E-Fast Sensitivity analysis of the Time-Delay Model

We now derive numerical simulations with $K_{C}=5482$ constant and $K_{C}$ periodic, like in the previous section. We choose as initial conditions $(E(0), A(0))=(0,50)$.

In Fig. 10, page 20, we illustrate the previous results when the carrying capacity $K_{C}$ is constant. When $\mathcal{R}<1$, mirids population decays till extinction and when $\mathcal{R}>1$, mirids population converge to a positive equilibrium.

As expected, when $t$ goes to infinity, the time-delayed model with constant parameters responds exactly like the non-delayed model, except that the threshold parameters is not exactly the same. When $\mathcal{R}<1(>1$ ), the system converges to the trivial (positive) equilibrium. In fact, with the same parameters, the deterministic model may converge to the positive equilibrium, while the time-delay model converges to the trivial equilibrium.

Figs. 11, page 21 and 12 , page 21 represent the time evolution of mirids with $K_{C}$ periodic and for different values for $C$, namely $C=5$ and $C=100$. When $\mathcal{R} \leq 1$, mirid population decays rapidly till extinction, whatever the values taken by $C$. In contrary, when $\mathcal{R}>1$, mirid population converges to a periodic solution, as expected, but with different amplitudes related ted 

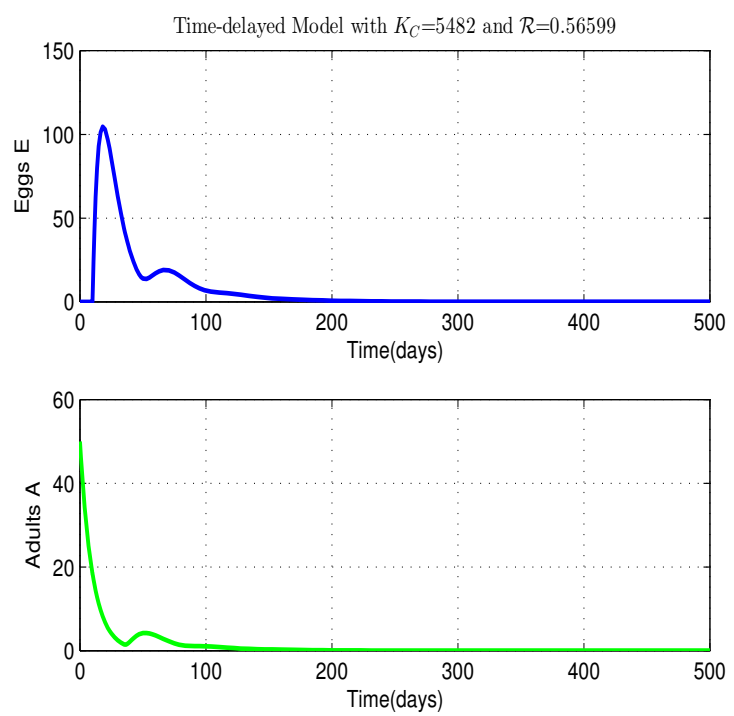

(a)
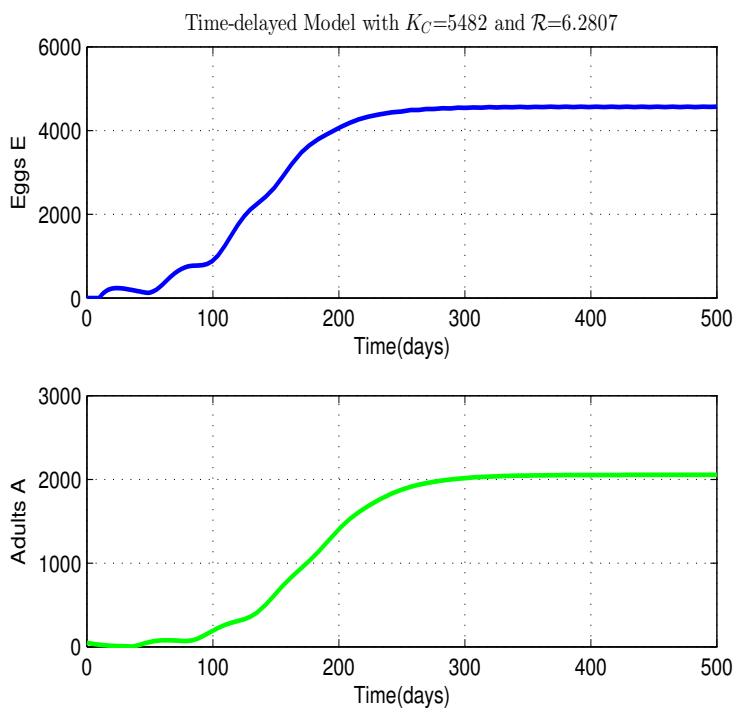

(b)

Figure 10: Time evolution of the Eggs and Adult Compartments for the time-delay model with constant parameters, $K_{C}=5482$, with: (a) $\mathcal{R}<1$

(b) $\mathcal{R}>1$

to $C$, indicating the importance of alternative resources in the maintenance and the size of the population. The simulations seem to be in good agreement with field observations. It seems also to be obvious that the removal of alternative resources should be part of control strategies to lower the impact of the mirids. Compared to the time-delayed model, the non-delayed model overestimates the population. Thus, even if from the mathematical point of view the long term behaviour is the same, the time-delayed model provides better estimate of the population size along the year than the non-delayed model.

We would like to emphasize that our study was very difficult because of the lack of population data. Many parameters values were estimated using raw data obtained in the field by Babin, in 2008, in Cameroon. We use these data to estimate several parameters like mortality of eggs, nymphs and adults $\left(\mu_{E}, \mu_{L}\right.$ and $\left.\mu_{A}\right)$, fecundity of adults female $(b)$, the mean duration of immature 

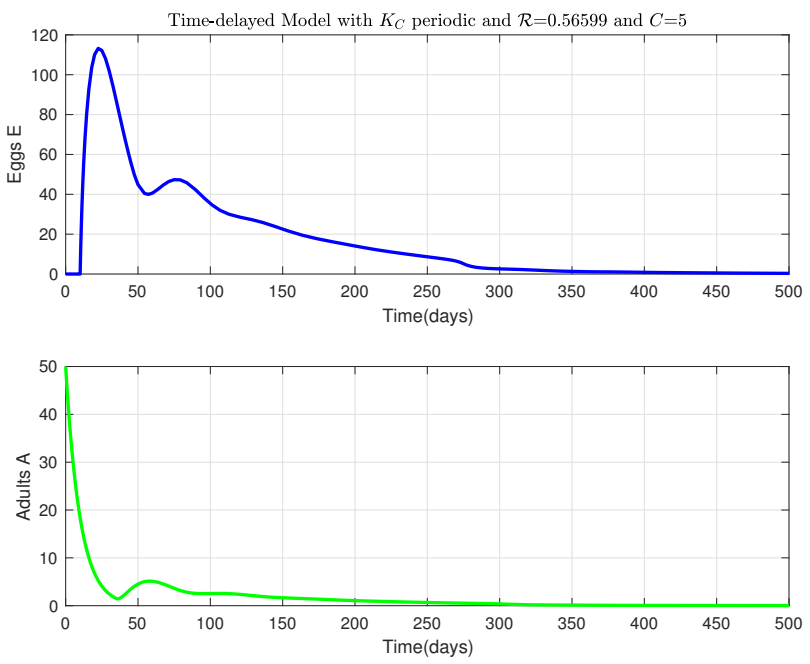
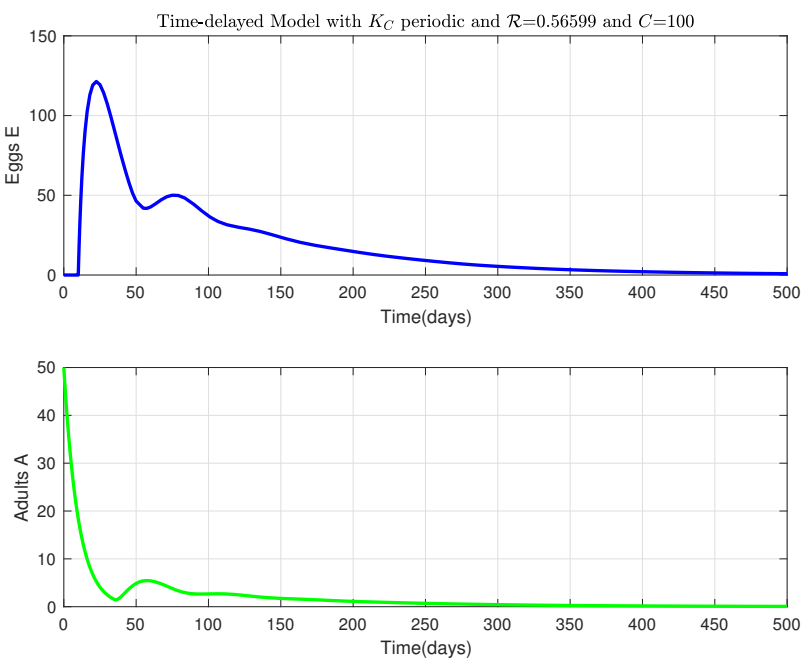

(a)

Figure 11: Time evolution of the Eggs and Adult Compartments for the time-delay model with constant parameters, $K_{C}$ periodic, such that $\mathcal{R}<1$, with:

(a) $C=5$

(b) $C=100$.
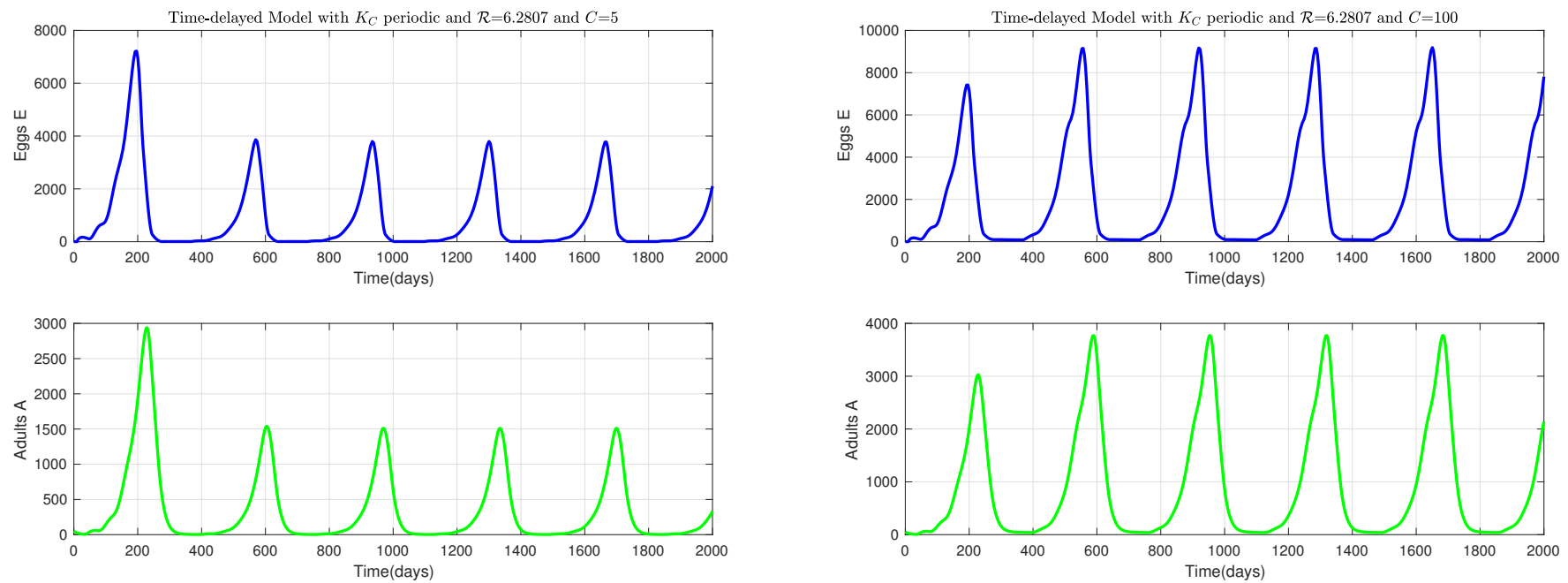

Figure 12: Time evolution of the Eggs and Adult Compartments for the time-delay model with constant parameters, $K_{C}$ periodic, such that $\mathcal{R}>1$, with:

(a) $C=5$

(b) $C=100$. 
females stage $\left(1 / \nu_{F}\right)$ and the mean necessary time for development of eggs. For some parameters (the sex-ratio $r$, the mean duration of egg stage $\left(1 / \nu_{E}\right)$ and the mean duration of nymph stage $\left.\left(1 / \nu_{L}\right)\right)$, we used data from [12] because it was the last experiment on $S$. singularis life cycle, realized in Cameroon. The most difficult data to obtain was the daily evolution of pods number in the plot. Using data from [16] and also based on our knowledge about the mean pods density per hectare, we construct our periodic function $K_{C}(t)$ which represent the daily appearance of pods and cherelles in the plot. However, mirids also feed and lay eggs on young shoots that lead to important damage able to cause the destruction of the tree over the years. It will be important to take into account this aspect and model the daily appearance of young shoots in the plot by a function $(S(t)$ for example). Then, it will be relevant to know the mirid frequency of feeding on host plants to have a good idea about the parameter $C$ because the time-evolution of mirids also depends on it considering that if $C$ is large, the level of mirid population increase. In our numerical simulations, we attributed to this parameter two values $C=5$ and $C=100$. According to these results, we suggest that it will be important to have several experiences in the field: to better estimate the daily appearance on pods and shoots in the plot, to have a good idea about the parameters of development of mirids and also to better estimate the parameter $C$.

\section{About Mirid Controls}

Several methods are used to control mirid population among which:

- cultural management, based on managing the system structure and composition to create unfavorable conditions for the development of mirids populations.

- varietal management, which consists in replacing the cacao varieties traditionally cultivated with more resistant and/or tolerant varieties to mirid attacks.

- chemical management, based on chemical insecticide used. This is the most widespread and efficient strategy to control mirid population in Cameroon. The chemical insecticides are applied twice to three times a year [10] (see also the CIRAD report published for cocoa producers.)

- semio-chemical management which consists of using synthetic sexual pheromone traps [35] which increases adult mortality (trapping), and prevents male insects finding females and mating (mating-disruption), and thus reduces the fecundity of female.

- last but not least, as showed in the previous simulations, a reduction of mirids alternative host (resources), i.e. decrease $C$, is also essential to have an efficient control.

Understanding the population dynamic of $S$. singularis is crucial for monitoring, forecasting and, then, controlling this pest population. Recent work in Ghana indicates (using the visual hand-height assessment method) that mirid populations (predominately nymphs) began to increase rapidly in April with an initial peak in May, followed by a rapid build-up in June [9]. In Cameroon, mirid /population is low on cacao from February to March. From June to July, the populations start to grow more or less rapidly depending on external conditions like weather and fruits production on the trees. The peak of the population appears between September and November when the pods are almost mature [12]. 
In Cameroon, mirids populations are mainly controlled by chemical insecticides. Three treatments are recommended per year: in June/July and August/September (propagation of mirids population); in November/December [25]. We will consider the impact of one treatment, two treatments and, finally three treatments. We will make a comparative study of treatments applied in systems with cacao only, $C=5$, and in agroforestry systems composed of cacao and associated trees that could be secondary resource for mirids, $C=100$.

According to the expert's knowledge and field obersvations, the insecticide has a decaying death rate over 8 weeks, that is summarized in Table 6 : .

\begin{tabular}{|c|c|c|c|c|c|c|c|c|c|c|}
\hline Time (in days) after the release & 1 & 3 & 8 & 16 & 24 & 32 & 40 & 48 & 56 & 60 \\
\hline Insecticide death rate & 1 & 1 & 0.9 & 0.2 & 0.1 & 0.05 & 0.025 & 0.01 & 0 & 0 \\
\hline
\end{tabular}

Table 6: Time dependent death-rate of chemical treatment

Remark 1 Synthetic insecticides like $\lambda$-cyhalothrine and Imidacloprid have a long residual effect, but it depends on several environmental factors, like rainfall. That is why Table 6 provides only a feedback from field experts according to the locations in Cameroon where these treatments have been studied and are already used.

We start our numerical simulations at the end of June, a period where the number of pods is increasing in the plot. We treat the plot respectively one, two, or three time(s) per year, as recommended to Cacao producers. We compare the efficacy between each treatment. The periods of treatment are given as follows:

- Treatment One with only one application per year: (beginning of) July ( $t=395,760,1125,1490,1855)$.

- Treatment Two with two applications per year: July $(t=395,760,1125,1490,1855)$ and September $(t=457,822,1187,1552,1917)$ (see [25], page 20).

- Treatments Three with three applications per year: July $(t=395,760,1125,1490,1855)$, September $(t=457,822,1187,1552,1917)$, and November $(t=518,883,1248,1613,1978)$ (see [25], page 20).

It is worth to mention that the periodicity of the two last treatments coincides with the duration of the chemical treatment after spreading. In Figs. 13, page 23, Fig. 14, page 23, and Fig. 15,page 24, we present the results obtained with the different treatments in two cases: $C=5$ and $C=100$. The case $C=5$ represents a full cacao crops, while $C=100$ may represent a cacao crop in an agro-forestry system, where additional resources (host trees) are available for mirids.

We summarize in Table 7, page 23 the impact of each treatment in the reduction of the wild population.

\begin{tabular}{|c|c|c|c|}
\hline & Treatment 1 & Treatment 2 & Treatment 3 \\
\hline$C=5$ & $74.8 \%$ & $92.9 \%$ & $97.8 \%$ \\
\hline$C=100$ & $58.7 \%$ & $84.8 \%$ & $94.1 \%$ \\
\hline
\end{tabular}

Table 7: Efficacy of each treatment - Percentage of reduction of the wild population 
As expected, 2 treatments are sufficient (more than $90 \%$ reduction of the population) when $C$ is small. An additional (third) treatment is particularly recommended when $C$ is large, i.e. $C=100$. These results are relevant with real observations in different type of plots, at least in Cameroon.
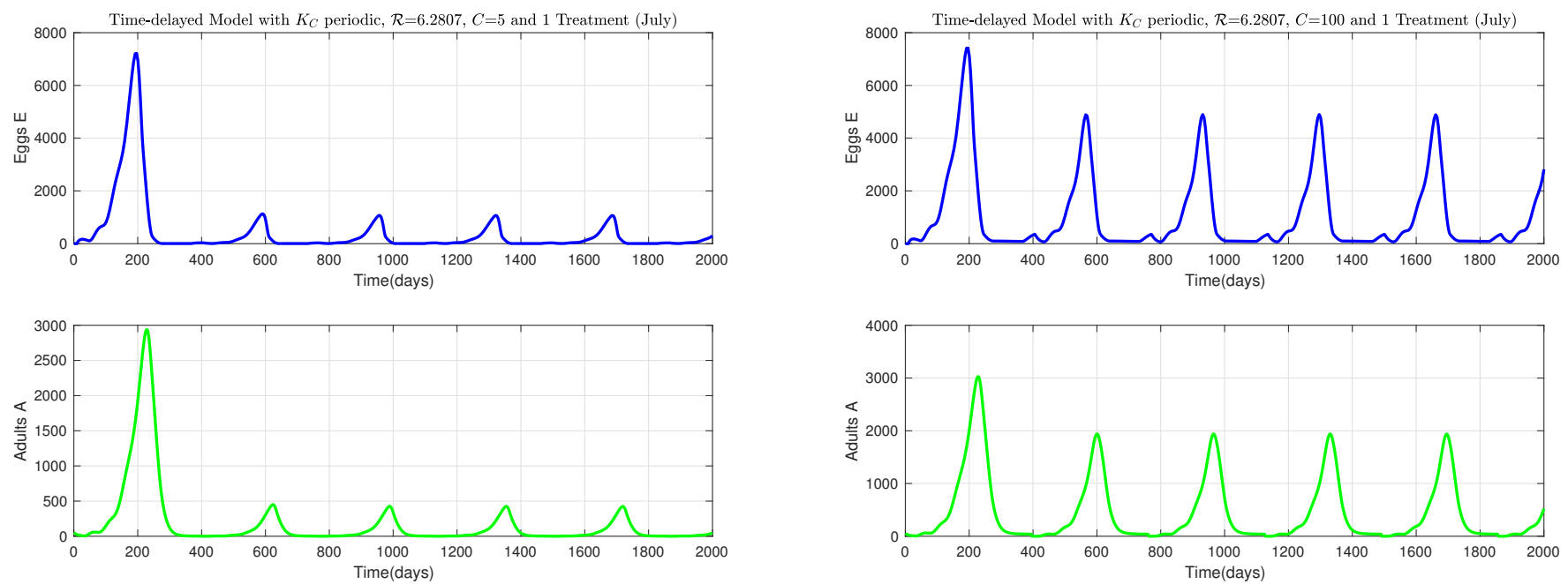

Figure 13: Time evolution of Mirids using only one treatment in the plot per year: (a) $C=5$, (b) $C=100$.
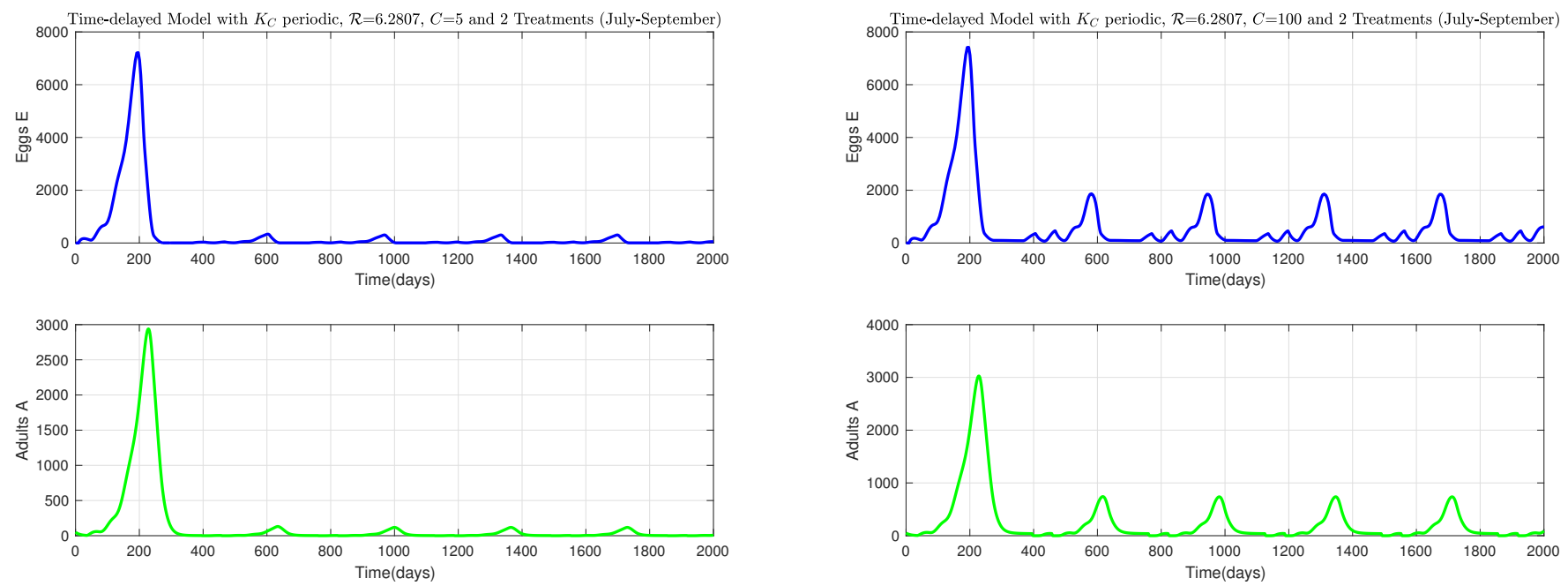

Figure 14: Time evolution of Mirids using two treatments per year in the plot: (a) $C=5$, $C=100$.

Although chemical insecticides are very efficient to control mirids, their recurrent use is widely questioned due to the immediate adverse effects that they cause in ecosystems via environmental pollution (impact non targeted species), induce resistance in the mirid population, and to the toxic effects on human health. In addition, these chemical products are very expensive. That is why, it could be more advantageous to consider sustainable control strategies, like for instance, mating disruption and trapping [35,41]. Mating disruption consists of introducing an artificial stimuli, 

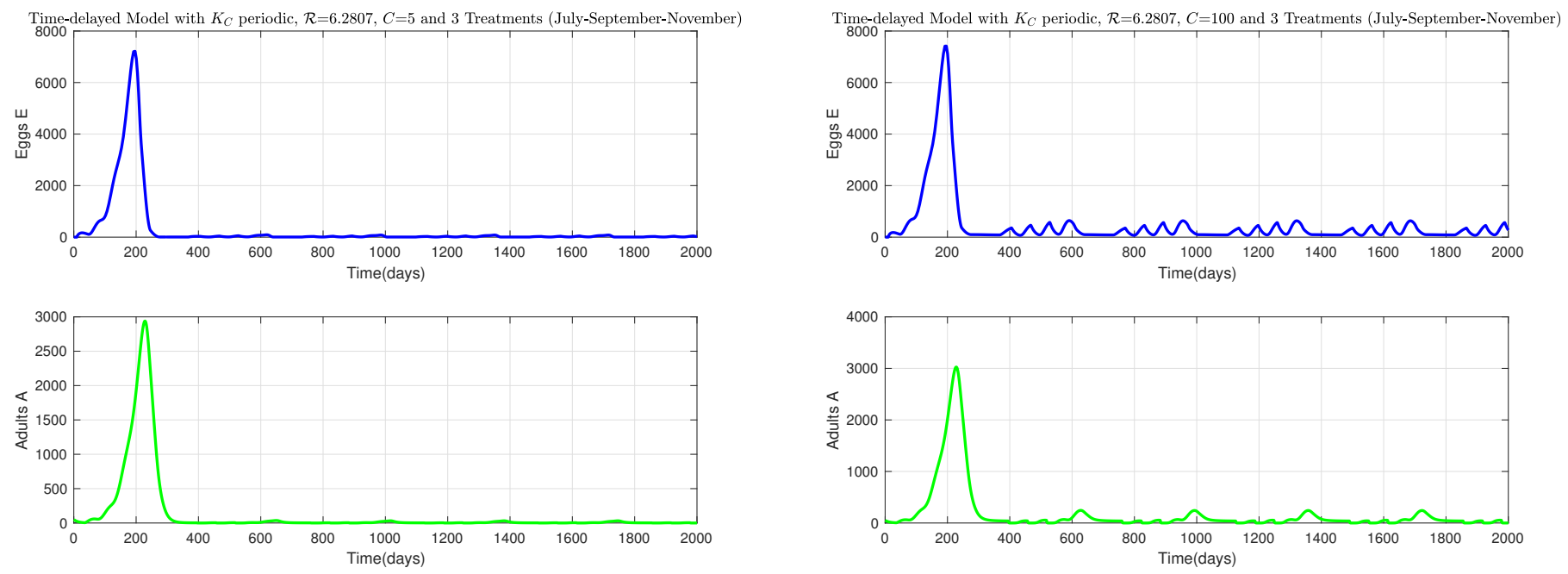

Figure 15: Time evolution of Mirids using three treatments per year in the plot: (a) $C=5$, (b) $C=100$.

like pheromones or para-pheromones, to confuse individuals, and, thus, disrupt mate localization, leading to long-term reduction of the population. In our case, we roughly assume that this implies a decrease of the female fecundity. Thus, for instance, if we reduce the daily female fecundity from 3.28 to 2 , the mirid population decreases: see Fig.16, page 24 .
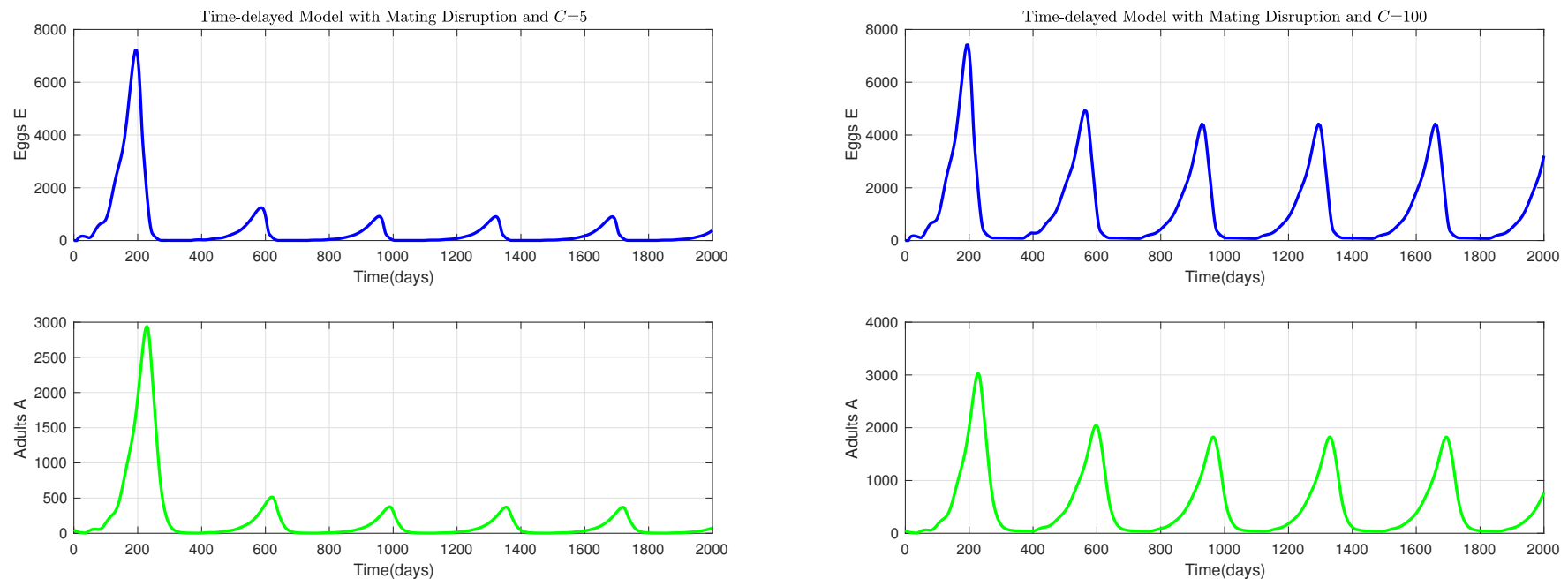

Figure 16: Time evolution of Mirids with control using mating disruption: (a) $C=5, \quad$ (b) $C=100$.

Another way to control mirids is the use of traps. Traps increase the adult mortality rate, $\mu_{A}$. For instance, if we increase the mortality $\mu_{A}$ from 0.07 to 0.1 , we observe a great reduction of the level of mirid population (see the time evolution of mirids in Fig. 17, page 25). According to the sensitivity analysis, the adult mortality $\mu_{A}$ is a sensitive parameter for the delayed model such that any increase may have a strong negative impact on the population.

In general, combining the two previous methods of control (mating disruption and trapping), 

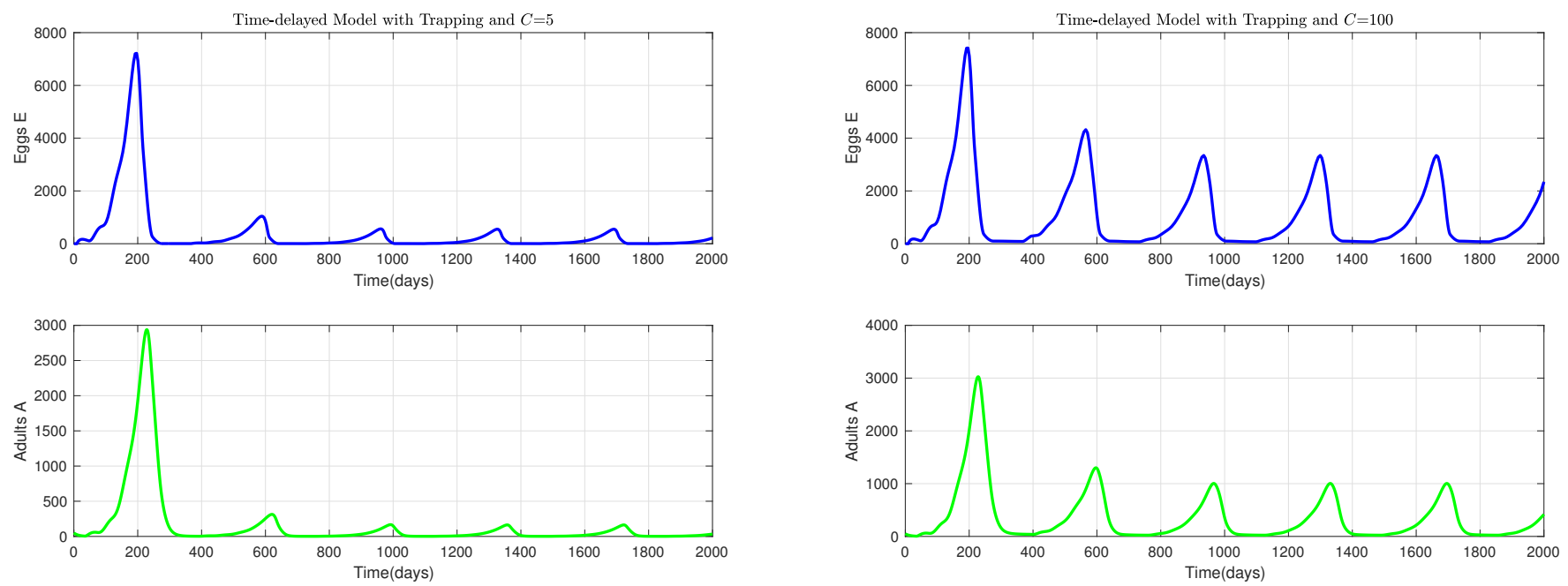

Figure 17: Time evolution of Mirids with control using trapping: (a) $C=5, \quad$ (b) $C=100$.

improve the previous results (see Fig. 18, page 26). This combination allows to reach a low level of population, right after the first year. . We summarize in table Table 8, page 25, the efficiency

\begin{tabular}{|c|c|c|c|}
\hline & Mating & Trapping & Trapping\&Mating \\
\hline$C=5$ & $70.5 \%$ & $85.5 \%$ & $96.5 \%$ \\
\hline$C=100$ & $49.4 \%$ & $71.0 \%$ & $90.1 \%$ \\
\hline
\end{tabular}

Table 8: Efficacy of Trapping, Matting, and Trapping-Mating - Percentage of reduction of the wild population

of each control methods. According to the given results, it seems possible to have a very efficient control of mirids without using chemical control. Clearly the combination of Mating and Trapping gives the best results whatever the values taken by $C$. This is in good agreement with recent field experiments [41].

In the previous simulations, we showed that a biological control strategy (using Mating and Trapping) can be a very good alternative to the use of insecticides. Last but not least, if it is possible, the reduction of alternative hosts in the plots is also an additional way to improve the efficacy of both controls.

\section{Conclusion}

In this paper, we studied the dynamics of a cocoa pest, mirids. From the best of our knowledge it is the first time that mathematical models are developed to study mirid pest. We first build a generic stage-structured ODE model to simulate the dynamic of the pest population considering the resource (available cocoa pods and additional tree hosts) as constant or as a periodic function. Our model enters the family of cooperative system, which facilitates its study. Thus, we show that there exists a threshold parameters, $\mathcal{N}_{0}$, also called the basic offspring number, that summarizes the dynamics of the system: when it is less than one, then the mirid population decreases till extinction; when it is greater than one, then mirids population persists. Then, based on the mirid's 

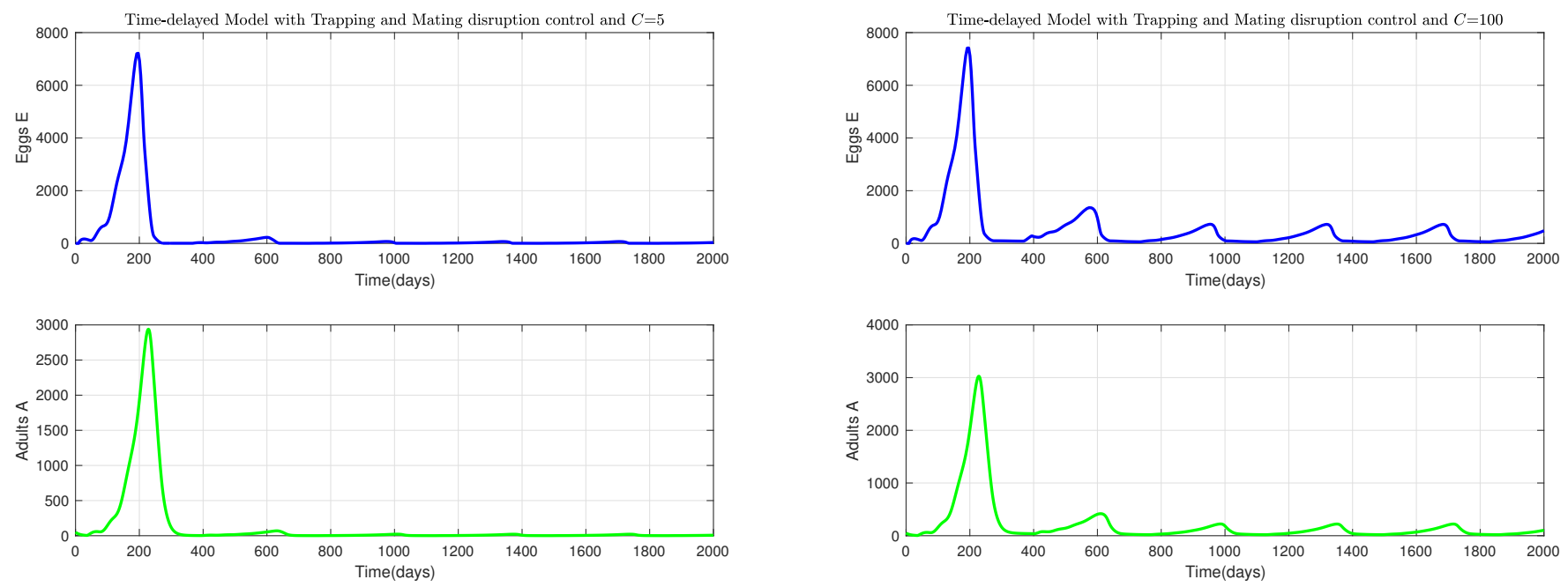

Figure 18: Time evolution of Mirids with control combining trapping and mating disruption; (a) $C=5, \quad(\mathrm{~b}) C=100$.

development stages and times, we also develop a cooperative delay model with two delays. We derive a theoretical study and estimate the related basic offspring number, $\mathcal{R}$. We illustrate the theoretical results through numerical simulations. We show that the outputs of the delayed system are more realistic than the non-delayed ODE model. We also highlighted that the presence of additional hosts can help the capsid population to maintain when pods are not available. Last but not least, for both models, we derive a sensitivity analysis that shows that the carrying capacity and the mortality rates are the most sensitive parameters.

Another objective of our work was to study ongoing mirid control strategies in Cameroon. We evaluate the impacts of chemical control and biological control (using mating disruption and trapping). Chemical control is used since a long time in Cameroon. It is very efficient when, at least, 2 treatments per year are applied. Our numerical simulations are in good agreement with the phytosanitary recommendations. However, we show that mating disrupting and trapping can be as efficient as chemical control, while being less detrimental to the environment. Of course, the reduction of additional hosts will improve the efficacy of the control.

According to these preliminary results, several ways of study are possible: (i) to propose to entomologists and agronomists to set up new experiments in the field in order to obtain additional and new data to improve our knowledge, to estimate some biological and ecological parameters in different environmental conditions, to better model the time dynamic of the mirids, and thus to improve their control, (ii) to develop a model where mating disrupting and trapping are effectively modeled, like in $[3,4]$, (iii) to couple our models to a pod growth model in order to take into account the impact of mirids on cocoa pods, (iv) to take into account the spatial distribution of mirids using a Metapopulation approach [24], or a Partial Differential Equation approach [23].

Acknowledgments: The first author is grateful to the DP "Agroforesterie Cameroun", CIRAD, IRD and INRIA-LIRIMA for logistical and financial support during the preparation and the finalization of this manuscript. 


\section{References}

[1] R. Adu-Acheampong, J. Jiggins, A. van Huis, A. Cudjoe, V. Johnson, O. SakyiDawson, K. Ofori-Frimpong, P. Osei-Fosu, E. Tei-Quartey, W. Jonfia-Essien, ET AL., The cocoa mirid (hemiptera: Miridae) problem: evidence to support new recommendations on the timing of insecticide application on cocoa in ghana, International Journal of Tropical Insect Science, 34 (2014), pp. 58-71.

[2] D. Alemagi, P. Minang, L. Duguma, A. Kehbila, F. Ngum, M. Noordwijk, O. Freeman, C. Mbow, J. de Leeuw, and D. Catacutan, Pathways for sustainable intensification and diversification of cocoa agroforestry landscapes in cameroon, Climate-Smart Landscapes: Multifunctionality in Practice. ASB Partnership for The Tropical Forest margins, (2014), pp. 347-359.

[3] R. Anguelov, C. Dufourd, and Y. Dumont, Mathematical model for pest-insect control using mating disruption and trapping, Applied Mathematical Modelling, 52 (2017), pp. 437457.

[4] - Simulations and parameter estimation of a trap-insect model using a finite element approach, Mathematics and Computers in Simulation, 133 (2017), pp. 47-75.

[5] R. Anguelov, Y. Dumont, and J. Lubuma, Mathematical modeling of sterile insect technology for control of anopheles mosquito, Computers and Mathematics with Applications, 64 (2012), pp. 374-389.

[6] R. Anguelov, Y. Dumont, and J. M.-S. Lubuma, On nonstandard finite difference schemes in biosciences, in Proceedings of the 4th International Conference on Application of Mathematics in Technical and Natural Sciences (AMiTaNS'11), M. Todorov, ed., vol. 1487, American Institute of Physics - AIP Conference Proceedings, 2012, pp. 212-223.

[7] J. C. Anikwé, A. A. Omoloye, F. A. J. C. Okelana, and R. Babin, Novel rearing technique, development biology, fecundity and morphometrics of the brown cocoa mirid Sahlbergella singularis haglung in nigeria, 2010.

[8] G. Awudzi, M. Asamoah, F. Owusu-Ansah, P. Hadley, P. Hatcher, and A. DayMOND, Knowledge and perception of ghanaian cocoa farmers on mirid control and their willingness to use forecasting systems, International Journal of Tropical Insect Science, 36 (2016), pp. 22-31.

[9] G. K. Awudzi, A. R. Cudjoe, P. Hadley, P. E. Hatcher, And A. J. Daymond, Optimizing mirid control on cocoa farms through complementary monitoring systems, Journal of Applied Entomology, 141 (2017), pp. 247-255.

[10] G. K. Ayenor, N. Roling, A. van Huis, B. Padi, and D. Obeng-Ofori, Assessing the effectiveness of a local agricultural research committee in diffusing sustainable cocoa production practices: the case of capsid control in ghana, International Journal of Agricultural Sustainability, 5 (2007).

[11] R. BABIN, Contribution à l'amélioration de la lutte contre le miride du cacaoyer Sahlbergella singularis Hagl. (Hemiptora: Miridae). Influence des facteurs agro-écologiques 
sur la dynamique des populations du ravageur., Thèse de Doctorat, Université Paul ValéryMontpellier III, biologie des populations et écologie, 2009.

[12] R. Babin, J. C. Anikwé, L. Dibog, and J. P. Lumaret, Effets of cocoa trees phenology and canopy microclimate on the performance of the mirid bug Sahlbergella singularis, Entomologia Experimentalis et Applicata, 141 (2011), pp. 25-34.

[13] R. Babin, B. Bisselua, L. Dibog, and J. Lumaret, Rearing method and life table data for the cocoa mirid bug Sahlbergella singularis haglund (hemiptera: Miridae)., Journal of Applied Entomology, 132 (2008), pp. 366-374.

[14] R. Babin, C. Fenoulllet, L. Legavre, T.and Blondin, C. Calatayud, A.-M. RisterucCI, AND M.-P. ChaPUis, Isolation and characterization of twelve polymorphic microsatellite loci for the cocoa mirid bug sahlbergella singularis, International journal of molecular sciences, 13 (2012), pp. 4412-4417.

[15] R. Babin, M. Gerben ten Hoopen, C. Cilas, F. Enjalric, Yede, P. Gendre, and J. P. Lumaret, Impact of shade on the spatial distribution of Sahlbergella singularis in traditional cocoa agroforests, Agricultural and Forest Entomology, 12 (2010), pp. 69-79.

[16] D. H. B. Bisselua, Yede, And S. Vidal, Dispersion models and sampling of cacao mirid bug Sahlbergella singularis (hemiptera: Miridae) on theobroma cacao in southern cameroon, Environmental Entomology, 40(1) (2011), pp. 111-119.

[17] J. Carayon, Caractères généraux des hémiptères bryocorinae, in Les mirides du cacaoyer, E. M. Lavabre, ed., IG-P. Maisonneuve et Larose, Paris, 1977, pp. 13-34.

[18] C. Collingwood, Biological control and relations with other insects, in Les mirides du cacaoyer, E. M. Lavabre, ed., IG-P. Maisonneuve et Larose, Paris, 1977, pp. 237-255.

[19] T. Davis, P. Kaufman, and D. Hogsette, J. Kline, The effects of larval habitat quality on aedes albopictus skip oviposition, J. Am. Mosq. Control Assoc., 31 (2015).

[20] F. De Abreu, M. Morais, S. Ribeiro, and A. Eiras, Influence of breeding site availability on the oviposition behaviour of aedes aegypti, Memorias Do Insti- tuto Oswaldo Cruz, 110 (2015).

[21] L. Dibog, R. Babin, A. Amang, J. Mbang, B. Decazy, S. Nyassé, C. Cilas, and A. B. Eskes, Effect of genotype of cocoa (theobroma cacao) on attractiveness to the mirid sahlbergella singularis (hemiptera: Miridae) in the laboratory, Pest Management Science, 64 (2008), pp. 977-980.

[22] E. Dounias and C. M. Hladick, Les agroforêts mvae et yassa du cameroun littoral : fonctions socioculturelles, structure et composition floristique, in Lálimentation en forêt tropicale : interactions bioculturelles et perspectives de développement, C. Hladik, A. Hladik, P. H., O. Linares, G. Koppert, and A. Froment, eds., UNESCO, 1996, pp. 1103-1126.

[23] C. Dufourd And Y. Dumont, Impact of environmental factors on mosquito dispersal in the prospect of sterile insect technique control, Computers and Mathematics with Applications, 66 (2013), pp. 1695-1715. 
[24] Y. Dumont, J. Soulie, And F. Michel, Modelling oil palm pollinator dynamics using two opposite approaches. some preliminary results., Mathematical Methods in Applied Sciences, to appear (2018), p. 22 pages.

[25] K. Edoh Adabe and E. NGO-Samnick, Production et transformation du cacao, in Collection PRO-AGRO, Ingénieurs Sans Frontières Cameroun (ISF Cameroun) et du Centre technique de coopération agricole et rurale (CTA), 2014.

[26] P. Entwiste, Insects and cocoa, in Cocoa, G.A.R.Wood and R.A.Lass, eds., Longman, London, 1985, pp. 366-443.

[27] P. Entwistle, Pests of Cocoa, Longman Group Ltd, London, 1972.

[28] D. Guest, Black pod: diverse pathogens with a global impact on cocoa yield, Phytopathology, 97 (2007), pp. 1650-1653.

[29] J. HALE, Theory of Functional differential equations, vol. 3 of Applied Mathematical Sciences, Springer, New York, 1977.

[30] J. JIFA, The algebraic criteria for the asymptotic behaviour of cooperative systems with concave nonlinearities, J. Syst. Sci. Complex., 6(3) (1993), p. 193.

[31] R. Kumar and A. Ansari, Biology, immature stages and rearing of cocoa-capsids (miridae: Heteroptera), Zoological Journal of the Linnean Society, 54 (1974), pp. 1-29.

[32] S. Laird, G. Awung, and R. Lysinge, Cocoa farms in the mount cameroon region: biological and cultural diversity in local livelihoods., Biodiversity and Conservation, 16 (2007), pp. 2401-2427.

[33] E. Lavabre, Recherches sur une méthode économique de contrôle des mirides du cacaoyer, Café Cacao Thé, 4 (1960), pp. 16-25.

[34] E. Lavabre, J. Decelle, And P. Debord, Recherches sur les variations des populations de mirides en côte d'ivoire, Café Cacao Thé, 6 (1962), pp. 287-295.

[35] R. Mahob, R. Babin, G. ten Hoopen, L. Dibog, Y. Yede, D. Hall, and C. Bilong BiLONG, Field evaluation of synthetic sex pheromone traps for the cocoa mirid, sahlbergella singularis(hemiptera: Miridae), Pest Management Science, 67 (2011), pp. 672-676.

[36] S. Marino, I. Hogue, C. Ray, and D. Kirschner, A methodology for performing global uncertainty and sensitivity analysis in systems biology, Journal of theoretical biology, 254 (2008), pp. 178-196.

[37] G. Matthews, T. Wiles, and P. Baleguel, A survey of pesticide application in cameroon, Crop Protection, 22 (2003), pp. 707-714.

[38] F. K. N'Guessan and N. Coulibaly, Dynamique des populations de mirides et de quelques autres déprédateurs du cacaoyer dans la région ouest de la côte d'ivoire., Proceedings of the 13th International Cocoa Research Conference, (2000), pp. 425-435. Cocoa Producer's Alliance, Malaysia. 
[39] F. K. N'Guessan, H. A. N'Guessan, P. W. N'Guessan, N. N'Dri Kouame, and Y. TANO, Variations saisonnires des populations de mirides du cacaoyer dans la région de l'indénié-djuablin en côte d'ivoire, Journal of Applied Biosciences, 83 (2014), pp. 7595-7605.

[40] B. Padi, G. Owusu, and N. Kumah, A record of desplatsia dewevrei (de wild \& th. dur.) (tiliales: Tiliaceae) as an alternative and potential breeding host plant for the cocoa mirid Sahlbergella singularis hagl., Actes de la $12^{\text {ème }}$ Conférence Internationale sur la Recherche Cacaoyère, San Salvador, Brésil, (1996), pp. 31-37.

[41] J. Sarfo, C. Campbell, and D. Hall, Design and placement of synthetic sex pheromone traps for cacao mirids in ghana, International Journal of Tropical Insect Science, (2018), pp. 110.

[42] H. Smith and M. Hirsch, Monotone dynamical systems, in Handbook of Differential Equations , Ordinary Differential Equations ( volume 2), A.Canada, P.Drabek, and A.Fonda, eds., Elsevier North-Holland, 2005, pp. 239-357.

[43] H. L. Smith, Cooperative system of differential equations with concave nonlinearities, Nonlinear Anal,: Theory, Meth. Appl., 10(10) (1986), pp. 1037-1052.

[44] — Monotone dynamical systems: An introduction to the theory of competitive and cooperative systems, American Mathematical Society, 41 (2008).

[45] D. Sonwa, A. Nkongmeneck, S. Weise, M. Tchatat, A. Adesina, and M. Janssens, Diversity of plants in cocoa agroforests in the humid forest zone of southern cameroon., Biodiversity and Conservation, (2007), pp. 2385-2400.

[46] G. Williams, Field observations on the cacao mirids, sahlbergella singularis hagl. and distantiella theobroma (dist.), in the gold coast. part i. mirid damage., Bulletin of Entomological Research, 44 (1953), pp. 101-119.

[47] Y. Yede, R. Babin, C. Djieto-lordon, C. Cilas, L. Dibog, R. Mahob, and C. F. Bilong Bilong, True bug (heteroptera) impact on cocoa fruit mortality and productivity, Journal of Economic Entomology, 4 (2012).

[48] A. YoudeoweI, The life cycles of the cocoa mirids Sahlbergella singularis hagl. and Distantiella theobroma dist., Nigeria Journal of Natural History, 7 (1973), pp. 217-223.

\section{Appendix A: Mains results of Cooperative Systems}

Here, we recall useful theorems (Theorem 3.1 in [43] and Theorem 5.5 in [30]).

Theorem .1 ([43], Theorem 3.1, page 1045$)$ Let $F(t, x)$ be continuous in $\mathbb{R} \times \mathbb{R}_{+}^{n}$, T-periodic in $t$ for fixed $x$. and assume $D_{x} F(t, x)$ exists and is continuous in $\mathbb{R} \times \mathbb{R}_{+}^{n}$. Assume that if $x \geq 0$, with $x_{i}=0$, then $F_{i}(t, x) \geq 0,1 \leq i \leq n, t \in \mathbb{R}$. Assume

$$
(M) \quad \frac{\partial F_{i}}{\partial x_{j}} \geq 0, i \neq j,(t, x) \in \mathbb{R} \times \mathbb{R}_{+}^{n}
$$

and

$$
D_{x} F(t, x) \text { is irreducible for each }(t, x) \mathbb{R} \times \mathbb{R}_{+}^{n}
$$




$$
(C) \text { if } 0<x<y \text {, then } D_{x} F(t, x) \geq D_{x} F(t, y) \text {. }
$$

Then every solution of (9) with $x\left(t_{0}\right) \geq 0$ can be continued to $\left[t_{0}, \infty\right]$ with $x(t) \geq 0$ for $t \geq t_{0}$.

$$
\text { If } F(t, 0) \equiv 0 \text { and }
$$

$$
z^{\prime}=D_{x} F(t, 0) z
$$

is the variational equation about $x \equiv 0$, them $\lim _{t \rightarrow \infty} x(t)=0$ for every solution of (9) with $x\left(t_{0}\right) \geq 0$ provided all Floquet multipliers of (19) lie inside or on the unit circle in the complex plane. If any multiplier of (19) lies outside the unit circle, then one of the following holds: (a) every solution $x(t)$ of (9) with $x\left(t_{0}\right) \geq 0$ satisfies $\lim _{t \rightarrow \infty} x(t)=\infty$, or (b) (9) possess a unique nonzero $T$-periodic solution $q(t)$. In the latter case $q(t)>0$ for all $t$ and $\lim _{t \rightarrow \infty} x(t)=q(t)$ for every solution of (9) with $x\left(t_{0}\right)>0$.

If $F(t, 0) \equiv 0$, then exactly one of the alternatives (a) or (b) occurs, except that $x\left(t_{0}\right)>0$ is replaced by $x\left(t_{0}\right) \geq 0$ above.

Let $A(t)$ be a $n \times n$ continuous matrix in $\mathbb{R} \tau$-periodic in $t$, denote

$$
\begin{aligned}
& \overline{a_{i j}}=\max _{0 \leq t \leq \tau} a_{i j}(t), \quad \underline{a}_{i j}=\min _{0 \leq t \leq \tau} a_{i j}(t) \\
& \bar{A}=\left(\overline{a_{i j}}\right), \quad \underline{A}=\left(\underline{a}_{i j}\right)
\end{aligned}
$$

Thus

$$
\underline{A} \leq A(t) \leq \bar{A}, \quad \text { for } \quad 0 \leq t \leq \tau .
$$

Let $p$ be a positive real number. In order to study system (8), we will use the following theorem:

Theorem .2 ([30], Theorem 5.5, page 203$)$ Let $F(t, x)$ be continuous in $\mathbb{R} \times \mathbb{R}_{+}^{n}$, T-periodic in $t$ for a fixed $x$ and assume $D_{x} F(t, x)$ exists and is continuous in $\mathbb{R} \times \mathbb{R}_{+}^{n}$ Assume that all solutions are bounded in $\mathbb{R}_{+}^{n}$ and $F(t, 0)=0$. Assume

- $\frac{\partial F_{i}}{\partial x_{j}} \geq 0, \quad(t, x) \in \mathbb{R} \times \mathbb{R}_{+}^{n}$

- and $A(t)=D_{x} F(t, 0)$ is irreducible for any $t \in \mathbb{R}$.

- If $0<x<y$, then $D_{x} F(t, x)>D_{x} F(t, y)$.

Then

1. If all principal minors of $-\bar{A}$ are non-negative, then $\lim _{t \rightarrow+\infty} x(t)=0$ for every solution of (9) in $\mathbb{R}_{+}^{n}$

2. If - $\underline{A}$ has at least one negative principal minor, then (9) possesses a unique positive T-periodic solution which attracts all initial conditions in $\mathbb{R}_{+}^{n}$. 